\title{
ESTUDIOS ETNOBOTÁNICOS V. NOMBRES DE PLANTAS Y SU UTILIDAD SEGÚN LOS MBYA GUARANÍ DE MISIONES, ARGENTINA
}

\author{
RAÚL NEREO MARTÍNEZ CROVETTO†'
}

\begin{abstract}
Summary: Martínez Crovetto, R. N. 2012. Ethnobotanical studies V. Plants names and their utility according the Guarani Mbya of Misiones, Argentina. Bonplandia 21(2): 109-133.

The meaning of the popular plant name is often related to the perception and the use given by certain societies to natural resources, so they provide valuable information about the importance of the environment for the human community that uses those names. Between the field notes of Martínez Crovetto we found an unpublished list of names and uses of plants that it is the result of his fieldwork with the Guarani Mbya of Misiones province, Argentina. In this edition of Bonplandia devoted to Ethnobotany we present these field notes by following the format of the series "Estudios etnobotánicos" published by the Ing. Agr. Martínez Crovetto.
\end{abstract}

Key words: Ethnobiology, phytonymy, useful plants, Misiones, unpublished field notes.

Resumen: Martínez Crovetto, R. N. 2012. Estudios Ethnobotánicos V. Nombres de plantas y su utilidad según los Mbya guaraní de Misiones, Argentina. Bonplandia 21(2): 109-133.

El significado de los fitónimos muchas veces está relacionando a la percepción y al uso que tiene determinada sociedad sobre los recursos naturales, aportando información valiosa acerca de la importancia del entorno para la comunidad que utiliza estos nombres de plantas. Entre las notas de campo del Ing. Agr. Raúl N. Martínez Crovetto se encontró una lista inédita de fitónimos y usos de plantas provenientes de su trabajo de campo con los guaraníes Mbya de la provincia de Misiones, Argentina, que se dan a conocer en esta contribución., siguiendo el formato de la serie "Estudios etnobotánicos" publicada por el Ing. Agr. Martínez Crovetto.

Palabras clave: Etnobiología, fitonimia, plantas útiles, Misiones, notas de campo inéditas

\section{Estructura del texto, signos fonéticos y aporte de los compiladores}

El listado de plantas ha sido estructurado del modo en que lo hacía Martínez Crovetto en su serie "Estudios Etnobotánicos", es decir, se presenta en primer lugar un listado alfabético de nombres guaraníes, su significado (entre paréntesis) y su correspondencia latina; en segundo lugar, un listado alfabético de nombres científicos, su equivalencia guaraní y sus usos.
Se ha agregado a la lista del segundo acápite las siglas de autores y la familia botánica. Asimismo, se consideró oportuno modificar los nombres guaraníes de las plantas registradas de acuerdo con las normas usuales en la actualidad. En este sentido, la grafía empleada concuerda con el diccionario de Guasch \& Ortíz (1995), con las siguientes consideraciones tomadas del diccionario Mbya guaraní- español de León Cadogan (1992): además de las cinco vocales orales se utiliza la $\boldsymbol{y}$ (i gutural), cinco vocales

${ }^{1}$ Instituto de Botánica del Nordeste, Casilla de Correo 209, 3400 Corrientes, Argentina. Recopilación realizada por Pirondo A. \& Keller H.A. 
nasales $(\tilde{\boldsymbol{a}}, \tilde{\boldsymbol{e}}, \tilde{\boldsymbol{\imath}}, \tilde{\boldsymbol{o}}, \tilde{\boldsymbol{u}})$ y la vocal $\tilde{\boldsymbol{y}}$ (i guto-nasal). La consonante $\boldsymbol{c h}$ tiene casi el mismo sonido que en inglés y en español. La consonante $\boldsymbol{j}$ es igual a la inglesa y a la catalana. Las consonantes $\boldsymbol{m}$, $\boldsymbol{m} \boldsymbol{b}, \boldsymbol{n}, \boldsymbol{n d}, \boldsymbol{n g}, \tilde{\boldsymbol{n}}, \boldsymbol{p}, \boldsymbol{r}, \boldsymbol{t}, \boldsymbol{v}$, como en español, pero no existe la $\boldsymbol{r} \boldsymbol{r}$. Las palabras guaraníes son mayormente agudas, por lo que se acentúan ortográficamente sólo aquellas que no lo son.

Se completó el epíteto específico de cierto número de especies que fueron anotadas por el autor en una larga lista detallada sólo hasta el nivel de género. Esta intervención se efectuó sólo para los géneros que en Misiones son monoespecíficos (Helietta, Heliocarpus, Leonurus, Osmunda), o que cuentan con unas pocas especies (Aspidosperma, Handroanthus, Sapium) cuyos nombres guaraníes han sido constatados en investigaciones posteriores. Respecto a las plantas listadas como indeterminadas, solamente se consideró agregar la identidad de aquellas cuyo fitónimo y usos son coincidentes y consistentes con investigaciones recientes (Keller, 2008).

En los cuadernos revisados no se han hallado referencias sobre el significado de los nombres guaraníes de las plantas anotadas por el autor, por lo que su traducción fue realizada por los compiladores.

\section{Índice de los nombres en lengua Mbya, su significado, y su equivalencia latina}

aguai (cuenta para sonajero), Chrysophyllum gonocarpum.

aguai chaiju (aguai amarillo), Pouteria sp. aguai guachu (gran aguai), Pouteria gardneriana.

aguape purũ'a (aguape hinchado), Eichhornia crassipes.

aguara nambi'i (orejita de zorro), Hydrocotyle bonariensis.

aguara po (mano de zorro), Abutilon sp., Galactia benthamiana, Galactia boavista.

aguara ruguái (cola de zorro), Schizachyrium paniculatum.

aguara ruguái mirĩ (pequeña cola de zorro), Bulbostylis juncoides.

aguara yva'i (fruto del zorro), Familia indet.

aguara'i ka'a (hierba del zorrito), Hybanthus parviflorus.

ãiño (solitaria), Cyperus sp. aju'y (genérico de Lauraceae), Nectandra megapotamica, Ocotea puberula.

aju'y guaika (laurel guaicá), Ocotea puberula.

aju'y ju (laurel amarillo), Ocotea diospyrifolia.

aju'y morochĩ (laurel blanco), Nectandra megapotamica.

aju'y ũ (laurel negro), Nectandra megapotamica, Ocotea diospyrifolia, Ocotea puberula.

aju'y vovõ (laure flojo), Prunus subcoriacea.

akarachĩ vai poã (medicina para cefalalgia intensa), Pistia stratiotes.

akarachy poã (medicina para cefalalgias), Smilax campestris.

amambái (nombre genérico de helechos), Dorypteris concolor, Familia indet. (Pteridophyta), Polypodium sp., Pteridium arachnoideum.

amambái typycha ovy (helecho escoba azul), Anemia phyllitidis var. tweediana.

amambái tyrẽ'y (helecho huérfano), Blechnum australe ssp. auriculatum.

amambái'i (pequeño helecho) Cheilanthes micropteris.

amba'y (árbol de la morada), Cecropia pachystachya.

amba’y guachu (grán amba’y), Schefflera morototoni.

amba'y guachu ka'aguy (grán amba’y de selva), Schefflera morototoni.

amba'y ka'aguy (amba'y de selva), Cecropia pachystachya, Schefflera morototoni.

amba'y'i (pequeño ama'y), Ricinus communis.

ambere ka'a (hierba de la lagartija ambere), Microgramma vacciniifolia, Stenachaenium riedelii.

ambere mbói (lagartija serpiente), Rhipsalis lumbricoides.

andai, Cucurbita moschata.

anguja chanjáu (sandía de ratón), Melothria cucumis, Melothria pendula, Melothria warmingii.

anguja ka'a (hierba del ratón), Evolvulus sericeus.

anguja ruguái (cola de ratón), Sida sp.

aña kygua (peine del demonio), Combretum fruticosum.

añangapiri mirĩ (pequeño añangapiri), Eugenia pyriformis var. uvalha.

añangapiri, Eugenia uniflora.

apere'a chanjáu (sandía de cuís); Melothria cucumis.

apere'a ka'a (hierba del cuís) Myrsine sp.

apyteryvi (textil y con duramen), Cordia trichotoma. 
aracha mirĩ (pequeño aracha), Psidium australe.

aracha ñu (aracha de campo), Psidium australe var. argenteum.

aracha, Psidium guajava, Psidium guineense, Psidium missionum.

araku ka'a (hierba del ave saracura), Begonia cucullata var. cucullata.

aratiku, Rollinia emarginata.

aratiku guachu (grán aratiku), Rollinia salicifolia.

avachĩ pichinga (híbrido con el español: "maíz pisingallo"), Zea mays.

avachĩ, Zea mays.

chamu'u mirĩ (pequeño palo borracho), Carica quercifolia.

chamu'u, Ceiba speciosa.

chandía (del español "sandía"), Citrullus lanatus ssp vulgaris.

chanjáu (del español "sandía"), Citrullus lanatus ssp vulgaris.

chanjáu rã (similar a la sandía), Wilbrandia ebracteata.

chanjáu víra (del español "sandía cambiada"), Cayaponia bonaerensis.

chapy’y (árbol del rocío), Dalbergia frutescens.

charandi ũ (sarandí negro), Ruprechtia salicifolia.

charandy morochĩ (sarandí blanco), Phyllanthus sellowianus.

chegrijo (del español “cedrillo"), Guarea guidonia. chevo'i (del español "cebolla"), Allium cepa.

chikoria mirĩ (híbrido con el español "pequeña achicoria"), Hypochaeris chillensis, Hypochaeris microcephala var. albiflora.

chikoria morochĩ (híbrido con el español "achicoria blanca"), Chaptalia sp.

chikoria'y (híbrido con el español “árbol de achicoria"), Senecio sp.

chimbo (ictiotóxico), Paullinia sp.

chimbo morochĩ (ictiotóxico blanco), Enterolobium contortisiliquum.

chimbo'y (árbol ictiotóxico), Enterolobium contortisiliquum.

chingachu ka'a (hierba del ave tingazú), Salvia sp.

chirka (de la vernácula “chilca”), Hyptis althaeifolia.

chivi ka'a (hierba del jaguar), Macfadyena unguiscati.

churuku'a ka'a (hierba del ave surucuá), Galphimia brasiliensis.

churuku'a ka'a guachu (gran hierba del ave surucuá), Begonia cucullata var. cucullata.

churukua ka'a ka'aguy (hierba silvestre del ave surucuá) Peperomia sp.

chypa cabaju (de la vernacular "cepa caballo"), Xanthium spinosum.

eira ka'a (hierba del mamífero irara), Heliotropium phylicoides.

eira viju ka'a (hierba del melipónido eira viju), Acanthospermum australe.

eiruchu ka'a (hierba de la abeja), Centratherum punctatum.

gajo ka'a ka'aguy (hierba silvestre del gallo), Cordia sp.

gajo ruguái (híbrido con el español "cola de gallo"), Vernonia sp.

gajo’y (híbrido con el español "árbol del gallo”) Vernonia sp.

guachu ka'a (hierba del venado), Desmodium affine, Passiflora sp., Rhynchosia lineata, Solanum sp.

guachu kara (ñame del venado), Dioscorea sp.

guachu kumanda (poroto de venado), Cassia bicapsularis, Chamaecristarepens, Desmanthus paspalaceus, Desmanthus tatuhyensis, Galactia striata var. striata, Senna pilifera var. pilifera.

guachu ky’iy yvyra (árbol ají del venado), Brunfelsia uniflora.

guachu ky’yĩ (ají de venado), Capsicum sp., Solanum bonariense, Solanum aff. chenopodioides, Vassobia breviflora.

guachu rembi'u (alimento de venado), Talinum racemosum.

guaimi rague (cabello de anciana) Selaginella sp.

guaja chimbo (ictiotóxico del guayá), Lonchocarpus muehlbergianus.

guajaki manduvi (maní del indígena Aché), Dicella nucifera.

guajayvi (textil del guayá), Cordia americana.

guajayvi mirĩ (pequeño guajayvi), Chomelia obtusa.

guajuvira morochĩ (guajayvi blanco), Terminalia triflora.

guajuvyra (deformación de guajayvi), Cordia americana.

guatambu, (contracción de "yvyra tambú": "madera con larvas"), Balfourodendron riedelianum.

guaviju mirĩ (pequeño guaviju), Psidium grandifolium.

guavira mirĩ (pequeño guabirá), Campomanesia aurea, Eugenia pyriformis var. pyriformis, Myrcia anómala, Myrcianthes pungens, Psidium australe var. argenteum.

guavira, Campomanesia xanthocarpa. 
guavirova, Campomanesia xanthocarpa.

guembe, Philodendron bipinnatifidum.

guey po (híbrido con el español "mano de buey"),

Bauhinia forticata ssp. pruinosa.

guyrapa rã (para hacer arcos), Casearia sp.

i'a rembo (fruto fálico), Lagenaria siceraria.

i’y pytãva (tallo acuoso y rojo), Begonia cucullata var. cucullata.

inambu jety (batata de la perdiz), Dioscorea $s p$., Oxalis conorrhiza.

inambu pytã ka'a (hierba del ave martineta), Bacharis pseudoteunifolia.

inambu ychypo (liana de la perdiz), Jacquemontia sp.

inga (fruto del árbol inga'y), Inga marginata, Inga verna.

inga guachu (gran ingá), Lonchocarpus muehlbergianus.

inga'y (árbol del inga), Inga marginata.

irague ua, Ambrosia elatior.

ita poty (flor de piedra), Lophophytum leandri.

jachy rapa (arco de la luna), Aspidosperma australe.

jachyta (caracol), Hydrocotyle exigua.

jachyta ka'a (hierba del caracol), Dichorisandra hexandra, Heliotropium sp., Hydrocotyle exigua, Hydrocotyleleucocephala, Tradescantia fluminensis

jachyta yrupe (tamiz de caracol), Hydrocotyle leucocephala.

jagua ka'a (hierba del perro), Glandularia sp., Lippia lippioides, Polypodium sp., Vernonia sp.

jagua petỹ (tabaco de perro), Vernonia $\mathrm{sp}$.

jagua pinda (anzuelo del jaguar), Macfadyena unguis-cati.

jagua pycha (dedos del pie del jaguar), Rhynchosia edulis.

jaguarata'y (tizón del jaguar), Styrax leprosus.

jaguarẽ ka'a (hierba del zorrino), Apium leptophyllum.

jaguarete py (pie de jaguar), Xanthium cavanillesii. jaguarundy guachu (gran jaguarundy), Piper sp.

jaguarundy, Piper sp.

jai rogue (hoja de maleza), Piper sp.

jakare ka'a (hierba del caimán), Anemia tomentosa. jaku ka'a (hierba de la pava de monte), Calea sp.

jaku pycha (dedo del pie de la pava de monte) Ipomoea $\mathrm{sp}$.

jaku rembi'u (alimento de la pava de monte), Celtis pubescens, Myrceugenia euosoma japu'a (fruto del ave boyero), Chloroleucon tenuiflorum.

jarakachĩ’a guachu (gran jarakachĩ’a), Jacaratia spinosa.

jarakati'a (fruto del árbol jarakati'a'y), Carica quercifolia.

jarakati'a'y (árbol del jarakati’a), Carica quercifolia.

jata'y (árbol del fruto jata), Butia yatay.

jate'i ka'a (hierba del melipónido jate'i), Achyrocline satureoides, Croton missionum.

jatevu ka'a (hierba de la garrapata), Euphorbia serpens, Ibicella lutea.

jatevu ka'a ñu (hierba de la garrapata del campo), Craniolaria argentina.

jayrogue rupe (maleza foliosa de escasa altura con base hinchada), Ocimun nudicaule.

jejy, Euterpe edulis.

jeruti ka'a (hierba de la paloma yerutí), Ruellia sp.

jety rã (similar a la batata), Ipomoea sp., Jacquemontia blancheti

jety ta'ytetu (batata de pecarí de collar) Spathicarpa hastifolia.

jety, Ipomoea batatas.

ju'a (espina frutal), Solanum bonariense.

ju'a para (espina con frutos variegados), Solanum aff. insidiosum.

ju'a pytã (espino de frutas rojas), Solanum sisymbriifolium.

ju'a'i (espino pequeño con frutos), Solanum pseudocapsicum.

ju'achy (espina que me hiere), Strychnos niederleini.

ju'achy'y (árbol de espinas que hieren), Celtis iguanaea.

ju'achy'y ka'a (árbol silvestre de espinas que hieren), Celtis iguanaea.

ju'aty (espino), Solanum sp.

ju'u (espina), Solanum aff. insidiosum.

ju'y ro (árbol de espinas ponzoñosas), Xylosma venosa.

jukery (espinoso), Acacia bonariensis.

jukery guachu (espinoso grande), Acacia recurva.

juya'a (fruto de juya), Solanum licopersicum.

jyva rachy ja (dueño de los males del brazo),

Dalbergia frutescens.

jyva'y (árbol del brazo), Dalbergia frutescens.

ka'a (hierba por antonomasia) Ilex paraguarienses.

ka'a arapachái (hierba del loro hablador), Agalinis genistifolia.

ka'a chiri, Ilex dumosa. 
ka'a ite (hierba verdadera), Solanum aff. chenopodioides.

ka'a mbara (planta discolor), Piptocarpha sellowi.

ka'a ovechĩ (planta de hojas blancas), Styrax leprosus.

ka’a porã (traducción del español "yerba buena"), Mentha rotundifolia.

ka'a rã (similar a la yerba), Citronella gongonha.

ka'a rẽ (hierba olorosa), Chenopodium ambrossioides.

ka'a rõ (planta de hoja), Mikania glomerata.

ka'a rupe (hierba de escasa altura con base hinchada), Ocimun nudicaule.

ka'a ruru (hierba hinchada), Amaranthus viridis, Talinum racemosum.

ka'a tái (hierba picante), Polygonum punctatum.

ka'a yna, Ilex dumosa.

ka'a yta (hierba diente), Miconia tristis.

ka'avo tory (yerba de la alegría), Vernonia sp.

ka'i aro (híbrido con el español "arroz de mono"), Pharus lappulaceus.

ka’i avachĩ (maíz de mono), Bilbergia sp., Tillandsia $\mathrm{sp}$.

ka'i karera (híbrido con el español "escalera de mono"), Bauhinia microstachya.

ka'i kygua (peine de mono), Amphilophium cynanchoides.

kachygua, (planta del cerrado), Trichilia catigua.

kaite mirĩ (pequeño kaite), Physalis viscosa.

kangorocha (del portugués "cangorosa": "cancerosa"), Maytenus ilicifolia.

kangue'i(huesito), Hyptisfloribunda, Iresinediffusa, Justicia sp., Poikilacanthus glandulosus.

ka'õvechingy (planta de hojas blanquecinas) Solanum mauritianum.

kapi'i (paja, pasto), Aristida riparia, Anthaenantia lanata, Axonopus compressus, A. suffultus, Carex sp., Chloris polydactyla, Digitaria sanguinensis, Eragrostis neesi, Elyonurus sp., Leptochloa virgata, Panicum sp., Paspalum coryphaeum, Paspalum nicorae, Paspalum plicatulum, Paspalum urvillei, Setaria sp., Setaria verticillata, Sporolobus indicus, Tridens brasiliensis.

kapi'i a (pasto con frutos), Coix lacryma-jobi.

kapi'i aimbe (pasto afilado), Cyperus sp.

kapi'i ãty (paja espinosa), Cenchrus equinatus.

kapi'i guachu (gran pasto), Erianthus trinii, Paspalum coryphaeum.

kapi’i kachĩ (pasto aromático), Kyllinga odorata.

kapi’i kuchuvirando Setaria sulcata. kapi'i pẽ (pasto petiso), Cynodon dactylon, Hippeastrum sp., Panicum repens, Paspalum conjugatum, Paspalum notatum.

kapi'i piri (pasto piri) Cyperus sp.

kapi'i poñy (pasto rastrero), Abildgaardia ovata, Cynodon dactylon, Paspalum notatum, Paspalum sp.

kapi'i pororo (pasto que explota al quemarse), Coleatania prionitis, Digitaria sp., Erianthus trinii.

kapi'i pororo ka'agüy (pasto reventón de selva), Pseudoechinolaena sp.

kapi'i pytã (paja roja), Andropogon lateralis, Andropogon sp.

kapi'i ravyju (pasto piloso), Elyonurus sp.

kapi'i viju (pasto piloso), Aristida pallens.

kapi'i'y (pasto árbol), Andropogon selloanus.

karachĩ (ñame blanco) Dioscorea sp.

karaguara (préstamo del quichua "calaguala"), Campyloneurum phyllitidis.

karaguata (bromelia) Eringium sp.

karaguata po'i (bromelia menuda), Eryngyum eburneum.

karaguata re'i (bromelia inservible), Pseudananas sagenarius.

karaguata, Bromelia balansae.

karaguata'i (pequeña bromelia), Eryngyum horridum, Eryngyum pristis, Tillandsia sp.

karaja ka'a (hierba del mono aullador), Commelina erecta var. erecta, Dichorisandra hexandra, Justicia sp.

karaja'y (árbol del mono aullador), Pterogyne nitens.

karanda, Copernicia alba.

karijo (del español "cadillo", "cardillo"), Cenchrus equinatus.

karijo yvate (híbrido con el español "cadillo alto"), Cenchrus myosuroides.

karito (del español "eucalipto"), Eucalyptus sp.

karova (rostro de la selva), Jacaranda micrantha.

karova guachu (gran karova), Aralia warmingiana.

karugua poã (medicina para el reumatismo), Cissampelos pareira.

karumbe ka'a (hierba de la Tortuga), Marchantia sp., Pontederia rotundifolia.

karuru mirĩ (pequña hierba hinchada), Portulaca oleracea.

kata, Ipomoea descolei.

katái (contracción de ka'a tái: "hierba picante"), Polygonum sp.

katu ka'a (buena planta), Mikania glomerata. 
katygua mirĩ (pequeño katygua), Trichilia elegans. katygua pytã (katygua rojo), Trichilia catigua. kavachingy (ictiotóxico de avispa), Ficus sp. kavachyngy'i (pequeño kavachingy) Solanum sp. kavara ka'a (hierba de la cabra), Eupatorium sp., Poiretia tetraphylla.

kavarin ruguái (híbrido con el españil "cola de jabalí"), Aristida riparia.

kavure'i ka'a (hierba del búho caburé), Oxalis sp.

kerandy rã (similar al kerandy), Aspidosperma australe.

kochi ka'a (hierba del pecarí labiado), Commelina erecta var. erecta.

kochi rembi'u (alimento del pecarí labiado), Tradescantia fluminensis.

koku pytã (pequeño koku), Allophylus guaraniticus.

koku, Allophylus edulis.

koto (algodón), Clematis bonaerensis, Clematis montevidensis var. montevidensis.

kravachita (del español "calabacita"), Cyclanthera hystrix.

krecheuma (préstamo del kaingange "que corta"), Chusquea ramosissima.

kuara pepẽ (fruto hueco lobulado), Cucurbita maxima.

kuarachi'a ka'a (hierba del picaflor ermitaño), Borreria poaya.

kuaray'a (fruto del sol), Luffa cylindrica.

kuchuvirando, Setaria sulcata.

kumanda choka (híbrido con el español: "poroto soja"), Glycine max.

kumanda ka'aguy (poroto de monte), Chamaecrista desvauxii var. mollisima.

kumanda yvyra'i (poroto arbolito), Cajanus cajan.

kumanda, Phaseolus vulgaris.

kumbari, Capsicum sp.

kuña poã (medicina para la mujer), Chevreulia sarmentosa.

kupa'y (árbol del kupa), Copaifera langsdorfii.

kurandyjo (del español "culantrillo"), Adiantum sp.

kurandyjo guachu (híbrido con el español "gran culantrillo"), Dorypteris concolor.

kurantrijo (del español "culantrillo"), Adiantum sp.

kuri'y (árbol del piñón) Araucaria angustifolia.

kurundy'y (árbol del kurundy), Trema micrantha.

kurunjy ũ mirĩ, (pequeño espécimen de Trema micrantha), Cheilanthes dichotoma,

kurunjy'ũ (kurunjy negro), Trema micrantha.

kurupa'y (árbol de corteza granulosa), Parapiptadenia rigida. kurupa'y morochĩ(kurupa'yblanco),Parapiptadenia rigida.

kurupa'y morochĩ (kurupa’y blanco) Albizia inundata.

kurupi ka'i (planta con la corteza granulosa), Sapium haematospermum.

kurupi ka'i guachu (grán kurupi ka'i), Sapium glandulosum.

kutolo ka'a (hierba del kutolo), Schinus terebenthifolius.

kyrỹ (tierno), Borreria poaya.

maino ka'a (hierba del colibrí), Justicia sp., Manettia inflata, Pavonia subrotunda.

maino rembi'u (alimento de colibrí), Justicia sp.

mandui (maní) Arachys hypogea.

mandui rã (similar al maní), Arachis glabrata var. hagenbeckii.

manduvi (maní) Arachys hypogea.

mandy'o, Manihot esculenta, Manihot grahami.

mandyju rã (similar al algodón), Xanthium cavanillesii.

mandyju, Gossypium herbaceum.

manjy'o, Manihot esculenta, Manihot grahamii.

marchera (del español "marcela"), Achyrocline satureoides.

mba'e pochy rova (rostro del demonio), Ibicella lutea.

mbaraka poã (remedio para guitarras), Catasetum fimbriatum, Oncidium biflorum.

mboguaju (espina del fantasma), Gleditsia amorphoides, Strychnos niederleini.

mbói ka'a (hierba de la serpiente), Cuphea linarioides.

mbóichy ka'a (hierba de la víbora mbóichy), Oxypetalum brachystemma.

mboka ja (dueño del proyectil), Acrocomia aculeata.

mborevy ka'a guachu (gran planta del tapir), Roupala montana. var. brasiliensis.

mbu'y (saeta), Conyza bonariensis, Leonurus sibiricus, Senecio brasiliensis, Senecio sp.

mbu'ychĩ (saeta blanca), Porophyllum ruderale. mbu'yrẽ (saeta hedionda), Porophyllum ruderale. mbu'ytĩ (saeta blanca), Porophyllum ruderale.

mburika ka'a (híbrido con el español "hierba de la mula"), Polypodium sp.

mburukuja mirĩ (pequeña pasionaria) Passiflora sp. mburukuja, Passiflora sp.

mburukuja'i (pequeña pasionaria) Passiflora sp.

merõ (del español "melón”), Cucumis melo.

moã katĩ (medicina olorosa), Croton urucurana. 
moã pyro’y, Sambucus australis.

moje'i (de la vernácula: “pequeño molle”), Schinus weinmannifolius.

naranka ka'aguy (híbrido con el español "naranja de la selva") Citrus $x$ aurantium.

naranka’y (árbol de la naranja), Citrus sp.

niño rupa (híbrido con el español "cama del niño), Aloysia gratissima var. sellowii, Lippia salviaefolia.

ñachĩ'u rã (similar a mosquito) Bidens subalternans, Osmunda regalis var. spectabilis.

ñandipa (deformación de ñandyta), Sorocea bonplandii.

ñandu apycha (oreja del ave ñandú), Campomanesia guazumifolia.

ñandu ka'a (hierba de la araña), Melochia ulmarioides.

ñandyta (el aro de nuestros canasos), Sorocea bonplandii.

ñerumi chĩ (ñerumi blanco) Bacharis pingraea .

ñerumi mirĩ (pequeño ñerumi), Ludwigia elegans.

ñerumi para (ñerumi overo) Baccharidastrum cfr. triplinervium, Croton sp.

ñerumi, Baccharis sp., Eupatorium sp.

ñu'aty (espino) Solanum sp.

ñuati pytã (espina roja), Solanum sisymbriifolium.

okarito (del español "eucalipto"), Eucalyptus sp.

paa guata (tranca el paso), Pistia stratiotes.

pakova de oro (híbrido con el español "banana de oro"), Musa sp.

pakova guachu (gran banano), Musa sp.

pakova karapẽ (banano enano), Musa sp.

pakuri, Rheedia brasiliensis.

pakuri vai (pakuri malo), Pouteria sp.

panamby ka'a guachu (gran hierba de la mariposa nocturna), Chamaecrista desvauxii var. mollisima.

para para'y, (árbol manchado), Jacaranda puberula.

paraicho (de la vernácula: "paraíso"), Melia azedarach.

pariparova guachu (gran pariparova) Piper sp.

pariparova, Piper sp.

paro chanto (del español "palo santo"), Trichilia clausseni.

paro chanto ka'aguy (híbrido con el español "palo anto de selva), Ambrosia elatior.

paroma ka'a (híbrido con el español "hierba de la paloma") Solanum sp.

pegua'õ (hoja de los bajos), Canna indica y otras especies del género, Hedychium coronarium,
Maranta sobolifera.

pekuru (superficie rugosa), Guadua trinii.

pengue ja (dueño de la pierna), Rhipsalis sp.

pengue poã (medicina para la pierna), Rhipsalis

lumbricoides.

perurija (préstamo de la vernácula "peludilla"),

Borreria sp., Gomphrena celosioides, Pfaffia

tuberosa, Richardia brasiliensis.

petỹ ra mirĩ (pequeña planta similar al tabaco),

Noticastrum macrocephalum.

petỹ, Nicotiana tabacum.

petỹrã (similar al tabaco), Vernonia sp.

pindo (que remata en un manojo de hojas), Syagrus romanzoffianum.

pindo rã (similar a la palmera pindo), Allagoptera campestris.

pindo rã (similar al pindó), Setaria sulcata.

pindo'i (pequeño pindo), Allagoptera campestris.

pipi, Petiveria alliacea.

pira ka'a (hierba del pez), Euphorbia papillosa, Eupatorium sp.

pira rembi'u (alimento del pez), Myrsine párvula.

piri, Cyperus entrerianus, Cyperus sp.

piri'i (pequeño piri), Cyperus sp.

pirinchoka'a(hierba delavepirincho), Centratherum punctatum.

pironko ka'a (hierba del pironko), Glandularia peruviana.

pitanga mirĩ (pequeña pitanga), Paramyrciaria delicatula.

pitanga, Eugenia uniflora.

poã ñu (medicina de campo), Chevreulia sarmentosa.

poã ro’y (medicina fría), Talinum racemosum.

poã ruvicha (cacique de las medicinas, catalizador), Casearia sylvestris.

popo ka'a (hierba de la mariposa), Phaseolus clitorioides.

poréo (de la vernacular "poleo"), Scoparia hassleriana.

poréo chiko (del español "poleo chico"), Bacharis pseudoteunifolia.

poréo'i (híbrido con el español "pequeño poleo), Hyptis floribunda.

pra pra'y (árbol manchado), Jacaranda puberula.

pykachu rembi'u (alimento de palomas), Casearia sylvestris, Chrysophyllum marginatum.

pykãu (préstamo del portugués “picão: picón”), Bidens subalternans.

pyky ka'a (hierba de la mojarra), Buchnera longifolia, Collaea stenophylla. 
pynõ (ortiga), Cnidoscolus albomaculatus.

pynõ guachu (gran ortiga), Cnidoscolus albomaculatus, Urera baccifera.

pỹnuarẽ'i ka'a (hierba del melipónido pỹnuarẽ'i), Vernonia brevifolia.

pyño (del español "pino") Araucaria angustifolia.

pyri, Tibouchina gracilis.

ryguachu ka'a (hierba de la gallina), Cissus sp.

ryguaja rembi'u (comida del pstácido ryguaja),

Schinus lentiscifolius, Schinus terebenthifolius,

Schinus weinmannifolius.

ta'ytetu manjy'o (mandioca del pecarí de collar), Wilbrandia ebracteata.

taa-taa (voy yendo), Desmodium incanum.

taguato ka'a (hierba del ave rapaz taguató), Hyptis mutabilis.

tajy chĩ (lapacho blanco), Cybistax antisyphilitica.

tajy morochĩ (lapacho blanco), Cybistax antisyphilitica, Handroanthus albus.

tajy ovy (lapacho celeste), Handroanthus heptaphyllus.

tajy ũ (lapacho negro), Handroanthus heptaphyllus.

tajy'i (lapachito), Scoparia hassleriana.

tajyja, Cayaponia bonaerensis.

takua (bambú, punta), Merostachys claussenii.

takua morochĩ (bambú blanco), Arundo donax.

takua rembo (bambú fálico), Chusquea ramosissima.

takua'y (bambú leñoso), Merostachys claussenii.

takuachĩ (bambú blanco), Arundo donax.

takuapi (bambú de corteza), Merostachys claussenii.

takuarẽ'ẽ (bambú dulce), Saccharum officinarum.

takuarembo'i (pequeño takuarembo), Olyra latifolia, Panicum sp.

takuaruchu (bambú grande), Guadua chacoensis.

takuary, Laciasis sp.

takuaty (población de bambú), Gynerium sagittatum.

tamonge guachu (gran soñolienta), Mimosa cruenata, Mimosa obstrigosa, Mimosa uliginosa, Mimosa velloziana.

tamonge guachu e'ỹ (soñolienta que no es grande), Mimosa polycarpa.

tamonge ju (mimosa amarilla), Chamaecrista repens.

tamonge pytã'i (mimosa tierna). Desmanthus paspalaceus, Desmanthus tatuhyensis.

tamonge'i (pequeña soñolienta), Mimosa paupera, Mimosa selloi

tapẽ kue (camino abandonado), Acanthospermum australe, Hyptis mutabilis, Iresine diffusa. tapi'a guachu (gran pene), Alchornea glandulosa. tapichĩ ka'a (hierba del conejo), Chamissoa altissima,

Psychotria carthagenensis, Sida urens.

tarami'ara, Tragia sp.

tarope, Maranta sobolifera.

taruma, Vitex sp.

tata jyva, Maclura tinctoria.

tata yva (fruto del fuego), Maclura tinctoria.

tatarẽ (fuego hediondo), Chloroleucon tenuiflorum.

tatu ruguái (cola de armadillo), Stachytarpheta australis

teju'i ka'a (hierba del lagarto), Eupatorium verbenaceum.

tembetary (de donde fluye el adorno labial) , Zanthoxylum sp.

tembetary chayju (tembetary amarillo), Zanthoxylum petiolare.

tembetary mirĩ (pequeño tembetary), Pterogyne nitens.

tembetary pytã (tembetary rojo), Zanthoxylum fagara.

tembetary ũ (tembetary negro), Zanthoxylum caribaeum.

tenke, Alsophylla setosa.

toro ka'a (hierba del toro), Buddleja stachyoides.

torypa (alegría total), Froelichia aff. paraguayensis, Vernonia sp.

tũ'a (híbrido con el español "fruto de tung"), fruto de Vernicia fordii.

tuja renapy'a (rodilla de anciano), Piper sp., Poikilacanthus glandulosus.

tukã revi kua rã (similar al ano de tucán), Calliandra foliolosa.

tuna (del español) Brasiliopuntia brasiliensis, Cereus sp.

tuna guachu (híbrido con el español "gran tuna"), Cereus stenogonus.

tupãchy kamby (leche de la madre del dios Tupã), Euphorbia serpens, Euphorbia sp.

tupãchy recha'i (ojos de la madre del dios Tupã), Dichorisandra hexandra, Tradescantia sp.

tuti'a, Solanum aff. insidiosum, Solanum sisymbriifolium.

tyevu poã (medicina para diarreas), Achyrocline satureoides.

tymba ju'y, Sebastiania brasiliensis.

typycha (escoba), Sida rhombifolia.

typycha ũ (escoba negra), Malvastrum coromandelianum, Myrcia bombycina, Sida rhombifolia. 
typycha’y (árbol de la escoba), Gomidesia palustris. u'yvarã, Cordyline spectabilis. ũapo’y (árbol de las raíces), Ficus sp. umby (sombra), Phytolacca dioica. uru ka'a (hierba del ave uru), Hydrocotyle exigua. uru ka'a (hierba del ave urú), Lippia lupulina,

Scoparia dulcis.

urunday, Astronium balansae.

urupe (tamiz), Craniolaria argentina.

urupe'i (tamiz pequeño), Pycnoporus sanguineus.

urutuka'a (hierba de la serpiente urutú), Centratherum punctatum.

vaka py (híbrido con el español "pie de vaca"), Bauhinia forticata ssp. pruinosa.

vaka pypore (híbrido con el español "pezuña de vaca"), Bauhinia forticata ssp. pruinosa.

vakũ pytã (pequeño vakũ), Allophylus guaraniticus. vakũ, Allophylus edulis.

vatatija ka'aguy (híbrido con el español "batatilla de la selva"), Alternanthera sp.

vervena guachu (híbrido con el español "verbena grande"), Verbena montevidensis.

vutia, Allagoptera campestris.

y'arã, Sicyos polyacanthus.

y'ary(capaz de flotar), Cedrela fissilis.

y'au (que viene del agua), Selaginella sp.

ychapy’y (árbol del rocío), Dalbergia frutescens.

ychau ka'a (hierba de la hormiga minera), Phyllantus sp., Poiretia tetraphylla.

ychongy, Leuhea divaricata.

ychypo (trepadora), Cayaponia bonaerensis, Gén. indet. (Bignoniaceae), Familia indet., Oxypetalum sp.

ychypo ava (liana del indígena ava), Dicella nucifera.

ychypo ju'u (trepadora espinosa), Smilax campestris.

ychypo ka'i karera (híbrido con el español "trepadora escalera de mono"), Bauhinia microstachya.

ychypo kamby (trepadora latescente), Araujia sericifera, Forsteronia sp.

ychypo mbói (liana de la serpiente), Convolvulus sp. ychypo mirombre (híbrido con el español "trepadra mil hombres"), Cissampelos pareira.

ychypo ñu (trepadora campestre), Ipomoea sp.

ychypo pẽ (liana aplanada), Bauhinia microstachya.

ychypo perẽ (trepadora con manchas), Cuphea glutinosa.

ychypo petái, Rhipsalis sp.

ychypo pytã (trepadora roja), Combretum fruticosum. ychypo ratã (trepadora dura), Combretum fruticosum.

ychypo rõ (liana de hoja), Mikania glomerata. ychypo timbo (liana ictiotóxica), Paullinia sp. ychypo ũ (liana negra), Paullinia elegans.

ychypo yvaro (trepadora yvaro), Arrabidaea mutabilis.

ychypo'y (liana acuosa), Mikania glomerata. yga'u (que viene del agua) Marchantia sp. ygary (capaz de flotar), Cedrela fissilis.

ygary mirĩ Cabralea canjerana.

ygary morochĩ (ygary blanco), Cedrela odorata.

ygary ũ (ygary negro), Cedrela fissilis.

ypeku'i ka'a (hierba del pájaro carpintero),

Stenandrium trinerve.

ypoty (flor), Ipomoea sp.

ypoty takua (flor espinosa), Xanthium cavanillesii.

yrupe (tamiz), Victoria cruziana.

yryvaja'y (árbol del psitácido yryvaja), Schinus terebenthifolius.

yryvu ka'a (hierba del jote) Oxypetalum sp.

yryvu retyma (canilla de jote), Zanthoxylum sp.

yva mirĩ (fruto pequeño), Schinus molle.

yva poõ (fruto gomoso), Melicoccus lepidopetalus.

yva purũ (fruto que estalla), Plinia trunciflora.

yva viju (fruto piloso), Myrcianthes pungens.

yva viju mirĩ (pequeño yvaviju), Campomanesia xanthocarpa.

yva’y (árbol de frutos), Schinus molle.

yvaei (fruto agrio), Hexachlamys edulis.

yvaei mirĩ (pequeño fruto agrio), Eugenia pyriformis var. pyriformis.

yvaporãity (abundante fruta buena), Plinia rivularis. yvaro (fruto tóxico), Prunus sp.

yvi (textil), Ceiba speciosa.

yvira (proveedor textil), Ceiba speciosa.

yvyra moã (árbol medicinal), Jacaranda micrantha.

yvoty (flor), Drymaria cordata, Glandularia peruviana, Stemodia verticillata.

yvoty ãiño (flor solitaria), Isostigma peucedanifolium.

yvy'a (planta tuberosa), Macfadyena unguis-cati. yvyra apytã (planta de frutos rojos), Rivina humilis. yvyra guachu (árbol grande), Phytolacca dioica. yvyra ita (madera de piedra), Astronium balansae. yvyra jakare (árbol del caimán), Erythrina cristagalli.

yvyra jepiro (árbol que se descorteza), Eugenia involucrata.

yvyra kachĩ (árbol hediondo), Lonchocarpus campestris, Lonchocarpus muehlbergianus. 
yvyra karai (árbol del dios Karai), Hennecartia omphalandra.

yvyra katu (árbol bueno), Xylopia brasiliensis.

yvyra ñechĩ, Balfourodendron riedelianum.

yvyra ñetĩ, Balfourodendron riedelianum.

yvyra ovy (árbol celeste), Helietta apiculata.

yvyra paje (árbol del hechizo), Myrocarpus frondosus.

yvyra pengy (madera quebradiza) Acacia poliphylla

yvyra pepẽ (árbol con contrafuertes) Holocalyx balansae.

yvyra petái (árbol de corteza picante), Pilocarpus pennatifolius.

yvyra pire chĩ (árbol de corteza blanca), Picrasma crenata.

yvyra pire morochĩ (árbol de corteza blanca), Picrasma crenata.

yvyra pire ũ (árbol de corteza negra), Heliocarpus popayanensis.

yvyra piriri (madera que chisporrotea), Sebastiania brasiliensis.

yvyra ponge (árbol del indígena kaingange), Dalbergia frutescens.

yvyra porã (árbol bueno) Diatenopteryx sorbifolia.

yvyra pororó (árbol reventón), Sebastiania brasiliensis.

yvyra pytã (madera roja), Peltophorum dubium.

yvyra pytã pyte (árbol de duramen rojo), Cabralea canjerana.

yvyra pytangy (madera rojiz), Aspidosperma polyneurom.

yvyra rapõ ju (árbol de raíz amarilla), Maytenus ilicifolia.

yvyra rei (árbol inservible), Cestrum sp.

yvyra ũ (madera negra), Actinostemon concolor.

yvyra u'u (árbol de la tos), Ruprechtia laxiflora.

yvyra yro añoty (planta picante que yo cultivo), Capsicum annum.

yvyra yvypy guachu (árbol de pie grande), Phytolacca dioica.

\section{Índice de nombres científicos, nombres en lengua Mbya y sus utilidades según las notas de Martínez Crovetto}

Abildgaardia ovata (Burm f.) Kral (Cyperaceae), kapi'i poñy. La raíz bien lavada ya macerada en agua fresca se da a los niños como febrífugo.

Abutilon sp. (Malvaceae), aguara po. Las hojas se aplican sobre forúnculos para secarlos.
Acacia bonariensis Gillies ex Hook. \& Arn. (Fabaceae), jukery. Se baña a las criaturas en la decocción de las hojas para que se duerman, tardando un par de horas en hacer efecto.

Acacia poliphylla DC. (Fabaceae), yvyra pengy.

Acacia recurva Benth. (Fabaceae), jukery guachu.

Las hojas se ponen en el tereré para calmar el dolor de apéndice.

Acanthospermum australe (Loefl.) Kuntze (Asteraceae), tape kue, eira viju ka'a. La decocción de la raíz se bebe como estomacal.

Achyrocline satureoides (Lam.) DC. (Asteraceae), marchera, tyevu poã, jate'i ka'a. La infusión de los capítulos se bebe como estomáquico y digestivo, la decocción de las hojas contra el dolor de vientre y la indigestión; en el mate para evitar enfermedades.

Acrocomia aculeata (Jacq.) Lodd. ex Mart. (Arecaceae), mboka ja. Comen el cogollo cocido, las frutas y las pepitas crudas y tostadas. Las pepitas tostadas y machacadas en el mortero, durante la luna nueva, se usa como crema para hacer crecer el cabello y el bigote.

Actinostemon concolor (Spreng.) Müll. Arg. (Euphorbiaceae), yvyra ũ. Las ramas se usan para hacer bastones pues se curvan con facilidad.

Adiantum sp. (Pteridaceae), kurantrijo, kurandyjo. La decocción de la planta se bebe en casos de diarrea y cefalalgias. Para este último fin se pica la planta en un mortero y luego se pone en la cabeza, atada con un trapo. También se emplea en el agua del mate.

Agalinis genistifolia (Cham \& Schltdl.) D' Arcy (Scrophulariaceae), ka'a arapachaí.

Albizia inundata (Mart.) Barneby \& J.W. Grimes (Fabaceae), kurupay morochĩ. La decocción de la corteza se usa para el lavado de heridas.

Alchornea glandulosa Casar (Euphorbiaceae), tapi'a guachu.

Allagoptera campestris (Mart.) Kuntze (Arecaceae), vutia. pindo rã, pindo'i. Comen las frutas.

Allium cepa L. (Liliaceae), chevo'i.

Allophylus edulis (A. St. -Hil., A. Juss. \& Cambess.)

Hieron. ex Niederl. (Sapindaceae), koku, vakũ. Comen las frutas, colocan las hojas y brotes en el tereré como refrescante estomacal y contra la tuberculosis. Para las enfermedades del corazón se prepara un remedio mezclando esta especie, perurija y verbena en $3 / 4$ litros de agua $\mathrm{y}$ haciendo hervir hasta dos litros. 
Allophylus guaraniticus ( A. St. -Hil.) Radlk. (Sapindaceae), koku pytã, vakũ pytã. Mismos usos que la anterior.

Aloysia gratissima var. sellowii (Briq.) Botta (Verbenaceae), niño rupa. La decocción de los brotes se bebe para limpiar el cuerpo de enfermedades internas y muy especialmente contra el dolor de cabeza.

Alsophylla setosa Kaulf (Cyatheaceae), tenke.

Alternanthera sp. (Amaranthaceae), vatatija ka'aguy. La decocción de la raíz se toma contra la tos.

Amaranthus viridis L. (Amaranthaceae), ka'a ruru. La decocción de la raíz se emplea en el lavado de heridas.

Ambrosia elatior L. (Asteraceae), paro chanto ka'aguy, iragüe ua. Las hojas en el mate se emplean contra la tos y la decocción en baño contra el pasmo.

Amphilophium cynanchoides (DC.) L. Lohmann (Bignoniaceae), ka'i kygua.

Andropogon lateralis Nees (Poaceae), kapi'i pyta. Mismo uso que Andropogon (especie indet.).

Andropogon selloanus ( Hack.) Hack. (Poaceae), kapi'i y.

Andropogon sp. (Poaceae), kapi’i pytã. La decocción de la raíz se toma en caso de purgaciones, debiendo beberse a razón de un litro por hora.

Anemia phyllitidis (L.) Sw. var. tweediana (Hook.) Hassl. (Schyzaceae), amambái typycha ovy. La decocción de la planta se usa como remedio fresco y en masajes para dolores del corazón.

Anemia tomentosa (Savigny) Sw. (Schizaceae), jakare ka'a. Se toma en el mate por las mañanas como tónico y para no sufrir enfermedades.

Anthaenantia lanata (Kunth) Benth. (Poaceae), kapi'i.

Apium leptophyllum (Pers.) F. Muell. (Apiaceae), jaguarẽ ka'a. La decocción en mate dulce o en té se emplea como bebida.

Arachis glabrata Benth. var. hagenbeckii (Harms ex Kuntze) F.J. Herm. (Fabaceae), mandui rã.

Arachys hypogea L. (Fabaceae), manduvi, mandui.

Aralia warmingiana (Marchal) J. Wen (Araliaceae), karova guachu. La decocción de la corteza se usa como purgante.

Araucaria angustifolia (Bertol.) Kuntze (Araucariaceae), pyño, kuri'y. Un puñado de corteza se hierve en un litro de agua, se le agrega un poco de azúcar y se pone en una botella que se entierra mirando al oeste; de esta bebida se toma una cucharada, aumentando la dosis cada día en una cucharada, no debiéndose comer carne fresca, picantes, ni tomar mate; cuando se concluya la botella se habrá curado de sus males. Comen las semillas tostadas, crudas o hervidas; molidas en un mortero, preparan el "sipa mbojape" (torta empastada con grasa y sal y tostada al rescoldo, o empastada con agua y cruda) y reviro mezclando la harina del piñón con harina de trigo y agua, grasa, sal y friendo la torta de ambos lados.

Araujia sericifera Brot. (Asclepiadaceae), ychypo kamby.

Aristida pallens Cav. (Poaceae), kapi'i viju. Las matas o fajas se usan como colchón para dormir.

Aristida riparia Trin. , kapi’i, kavarin ruguái. La decocción de las inflorescencias se las da a los enfermos graves para curarlos.

Arrabidaea mutabilis Bureau \& K. Schum. (Bignoniaceae), ychypo yvaro. Una vez lavada la cabeza en el arroyo se frotan con las hojas para curar cefalalgias.

Arundo donax L. (Poaceae), takua morochĩ, takuachĩ. Se usa para techados, astil de flechas, y para cestas y canastos.

Aspidosperma australe Müll. Arg. (Apocynaceae), jachy rapa, kerandy rã.

Aspidosperma polyneurom Müll. Arg. (Apocynaceae), yvyra pytangy.

Astronium balansae Engl. (Anacardiaceae), urunday, yvyra ita. La corteza en decocción se recomienda como remedio.

Axonopus compressus (Sw.) P. Beauv. (Poaceae), kapi'i.

Axonopus suffultus (J.C.Mikan ex Tin.) Parodi (Poaceae), kapi'i.

Baccharidastrum cfr. triplinervium (Less.) Cabrera (Asteraceae), ñerumi para. La decocción de la planta en sal se usa para el lavado de heridas.

Bacharis pingraea D.C. (Asteraceae), ñerumi chĩ La decocción de la planta en sal se usa para el lavado de heridas. Las hojas secas y pulverizadas sirven para curar quemaduras.

BacharispseudoteunifoliaI.L.Teodoro(Asteraceae), inambu pytã ka'a, poréo chiko. La decocción se bebe cuando el cuerpo está dolorido. Las mujeres la toman luego del parto, a razón de medio litro por hora, para atenuar dolores.

Baccharis sp. (Asteraceae), ñerumi. Escobas rústicas. 
Balfourodendron riedelianum (Engl.) Engl. (Rutaceae), guatambu, yvyra ñechĩ, yvyra ñetĩ. Las hojas machacadas en sal y molidas se emplean como hemostático en caso de cortaduras. La decocción de la corteza en lavados, sirve para lavar las llagas bucales de los niños.

Bauhinia forticata Link ssp. pruinosa (Vogel) Fortunato \& Wunderlin (Fabaceae), vaka py, guey po, vaka pypore. Las hojas puestas en agua para el mate se recomiendan para las cefalalgias y el reumatismo. La decocción con sal se usa para el lavado de granos.

Bauhinia microstachya (Raddi) J.F. Macbr. (Fabaceae), ka'i karera,ychypo ka'i karera, ychypo pẽ. La decocción de las hojas se bebe para los dolores hepáticos. Para purificar la sangre bebe la decocción a razón de tres cucharadas antes de las comidas. Trozos de palo machacado se arrojan al agua para embarbascar peces; si la dosis es suficiente, estos mueren en una hora.

Begonia cucullata Willd. var. cucullata (Begoniaceae), churuku'a ka'a guachu, araku ka'a, i’y pytava. Las hojas machacadas y puestas en agua fría, lo mismo que el jugo de los tallitos, sirve para el lavado de ojos en caso de conjuntivitis y como bebida contra la acidez estomacal.

Bidens subalternans DC. (Asteraceae), pykãu, ñachĩ'u rã. La decocción se emplea en lavados en casos de conjuntivitis y como bebida cuando el parto se retrasa; la maceración de los capítulos en agua fría, en forma de gotas tres veces al día, se usa en la conjuntivitis.

Bilbergia sp. (Bromeliaceae), ka’i avachĩ.

Blechnum australe L. ssp. auriculatum (Cav.) de la Sota (Blechnaceae), amambái tyre'y. Las mujeres para no tener hijos colocan este helecho mezclado con corteza de lapacho en el agua para el mate y beben cada mes. La maceración de las hojas en agua fría se toma para el dolor de cabeza.

Borreria poaya (A.St. -Hil.) DC. (Rubiaceae) kyrỹ, kuarachi ka'a. El té de la planta se bebe contra la fiebre. La decocción tibia, en bebida y lavados, se usa como calmante para dolores reumáticos.

Borreria sp. (Rubiaceae), perurija. La decocción se bebe contra cualquier enfermedad interna.

Brasiliopuntia brasiliensis (Willd.) A. Berger
(Cactaceae), tuna. Para activar la circulación de la sangre, cortan los cladodios en rodajas que se ponen en agua fría y se dejan en maceración durante la noche, y a la mañana se friccionan el cuerpo y las piernas; comen las frutas.

Bromelia balansae Mez. (Bromeliaceae), karaguata. Consumen las frutas cocidas en agua, esta se recomiendan contra las vermes intestinales. La decocción del rizoma, usada como agua para el mate, se recomienda para el dolor de cintura. De las fibras del mismo se hacen sogas.

Brunfelsia uniflora (Pohl) D. Dom. (Solanaceae), guachu ky'iy yvyra. La fruta picada se usa como condimento para las comidas.

Buchnera longifolia Kunth (Scrophulariaceae), pyky ka'a. La decocción de las hojas, enfriada, se da a los niños contra la diarrea.

Buddleja stachyoides Cham. \& Schltdl. (Buddlejaceae), toro ka'a. Se toma la decocción de la planta tomada junto con tape kue y jate'i ka'a, para calmar el dolor de pecho en caso de ataques violentos de tos.

Bulbostylis juncoides (Vahl) Kük.ex Herter (Cyperaceae), aguara ruguái mirĩ. Se usa la planta para hacer chorizos de barro para paredes de rancho.

Butia yatay (Mart.) Becc. (Arecaceae), jata'y.

Cabralea canjerana (Vell.) Mart. (Meliaceae), yvyra pytã pyte, ygary mirĩ. La decocción de la corteza se toma para dolores estomacales y cefalalgias.

Cajanus cajan (L.) Millsp. (Fabaceae), kumanda yvyra'i. Cultivan esta especie para aprovechamiento de las semillas que consumen hervidas.

Calea sp. (Asteraceae), jaku ka'a.

Calliandra foliolosa Benth. (Fabaceae), tukã revi kua rã.

Campomanesia aurea O. Berg. (Myrtaceae), guavira mirĩ. Comen las frutas.

Campomanesia guazumifolia (Cambess.) O. Berg. (Myrtaceae), ñandu apycha. Las hojas se ponen en el mate para calmar el dolor de barriga. La decocción de las mismas o de la corteza se emplea en el lavado de los oídos para curar la sordera. Se comen las frutas y se recomienda para eliminar vermes intestinales.

Campomanesia xanthocarpa O. Berg. (Myrtaceae), guavira, guavirova, yva viju mirĩ. La decocción de las hojas se emplea como bebida contra el chevo'i (vermes intestinales), con las hojas 
machacadas y puestas en agua fría, obtienen un digestivo. Con la decocción de las hojas calman el vómito de los niños. Comen las frutas.

Campyloneurum phyllitidis (L.) C. Presl (Polypodiaceae), karaguara. La decocción de las raíces se bebe tibia al acostarse y levantarse, en caso de tos convulsa.

Canna indica L. (Cannaceae) y otras especies del género, pegua'õ. La decocción de las raíces es tomada mensualmente a razón de un litro cada hora, por las mujeres que no quieren tener más hijos.

Capsicum annum L. (Solanaceae), yvyra yro añoty. Capsicum sp. (Solanaceae), guachu ky’yĩ, kumbari. Usan las frutitas como condimento de comidas.

Carex sp. (Cyperaceae), kapi'i.

Caricaquercifolia(A.St.-Hil.)Hieron(Caricaceae), jarakati'a, jarakati'a'y, chamu'u mirĩ. Comen la fruta.

Casearia sylvestris Sw. (Flacourtiaceae), pykachu rembi’u, poã ruvicha. La decocción de la corteza es considerada como preventiva contra cualquier clase de enfermedades

Casearia $s p$. (Flacourtiaceae), guyrapa rã. Con la madera fabrican arcos de caza.

Cassiabicapsularis L. (Fabaceae), guachu kumanda. La decocción de las hojas se bebe para limpiar el cuerpo de enfermedades.

Catasetum fimbriatum (C. Morr.) Lindl.\& Paxton (Orchidaceae), mbaraka poã. Mismo uso que Oncidium bifolium.

Cayaponia bonaerensis (Mill.) Mart. Crov. (Cucurbitaceae), ychypo, chanjáu vira, tajyja. La decocción de la raíz se recomienda para el dolor de vientre.

Cecropia pachystachya Trécul (Cecropiaceae), amba'y, amba'y ka'aguy. La decocción de los cogollos se bebe contra la tos. Con la misma bebida pero cosechados los cogollos en luna nueva, se enjuaga la boca en forma prolongada durante dos o tres días, para voltear los dientes. O bien, se prepara un jarabe con azúcar quemada contra la tos.

Cedrela fissilis Vell. (Meliaceae), ygary, y'ary, ygary ũ. Con las hojas maceradas en agua fría se prepara una bebida refrescante.

Cedrela odorata L. (Meliaceae) ygary morochĩ.

Ceiba speciosa (A.St.-Hil., Juss. \& Cambess.) Ravenna (Bombacaceae), yvira, yvi, chamu'u. Con las fibras de la semilla se fabrica hilo que se emplea para asegurar las puntas de las flechas, con la corteza retorcida hacen sogas.

Celtis iguanaea (Jacq.) Sarg. (Celtidaceae), ju'achy'y ka'a, ju'achy'y. Las hojas puestas en el mate se usan como refrescante y contra las fiebres gripales, comen las frutas.

Celtis pubescens (Humb. \& Bonpl.) Spreng. (Celtidaceae), jaku rembi'u. Comen las frutas.

Cenchrus equinatus L. (Poaceae), kapi'i ãty, karijo. La decocción de las raíces se toma para purificar la sangre.

Cenchrus myosuroides Kunth (Poaceae), karjo yvate. Mismo uso que la anterior.

Centratherum punctatum Cass. (Asteraceae), urutu ka'a, pirincho ka'a, eiruchu ka'a. Mezclado con Bacharis dos alas, para calmar el dolor de estómago. La decocción se usa en baños contra el reumatismo.

Cereus stenogonus K. Schum. (Cactaceae), tuna guachu. Comen las frutas, los extremos de los cladodios se maceran en agua fría y se toma contra la fiebre. Cortados en trozo y mezclados con grasa de lagarto, se dan a los perros cuando sangran por las orejas.

Cereus sp. (Cactaceae), tuna. Mismos usos que $C$. stenogonus.

Cestrum sp. (Solanaceae), yvyra rei.

Chamaecrista desvauxii (Collad.) Killip var. mollisima (Benth.) H. S. Irwin \& Barneby (Fabaceae), panamby ka'a, guachu kumanda ka'aguy. La decocción se bebe contra la tos.

Chamaecrista repens (Vogel) H.S. Irwin \& Barneby (Fabaceae), tamonge ju, guachu kumanda. La decocción de la planta tiene virtudes estomacales.

Chamissoaaltissima(Jacq.)Kunth(Amaranthaceae), tapichi ka'a.

Chaptalia sp. (Asteraceae), chikoria morochĩ.

Cheilanthes dichotoma Sw. (Pteridaceae), kurundy ju mirĩ. La decocción de la planta se usa como remedio fresco y para dolores del corazón.

Cheilanthes micropteris Sw. (Pteridaceae), amambái'i. Mismos usos que Aneimia phyllitidis (Schyzaceae).

Chenopodium ambrossioides L. (Chenopodiaceae), ka'a rẽ. Las hojas maceradas en agua tibia se dan contra el empacho de los niños. La decocción de raíces, hojas y tallos contra las lombrices intestinales.

Chevreulia sarmentosa (Pers.) S.F. Blake (Asteraceae), kuña poã, poã ñu. Las mujeres 
comen la planta en caso de menstruaciones excesivas.

Chloris polydactyla (L.) Sw. (Poaceae), kapi'i. Usan la planta para techar ranchos.

Chloroleucon tenuiflorum (Benth.) Barneby \& J.W. Grimes (Fabaceae), japu'a, tatarẽ. La decocción de las hojas en el mate, o directamente se toma para la gripe.

Chomelia obtusa Cham. \& Schltdl (Rubiaceae), guajayvi mirĩ. El agua fría con las hojas mezcladas se emplea en lavados contra el dolor de vista.

Chrysophyllum gonocarpum (Mart. \& Eichler) Engl. (Sapotaceae), aguai. Comen las frutas hervidas. La decocción de la corteza se toma contra la debilidad.

Chrysophyllum marginatum (Hook. \& Arn.) Radlk. (Sapotaceae), pykachu rembiu. Consumen las frutitas.

Chusquea ramosissima Lindm. (Poaceae), takua rembo, krecheuma (kaingange). Usan las cañas para armazón de cestos. Con la corteza de los entrenudos, cortada en tiras, atan las puntas de las flechas. Las cañas quemadas y calientes se emplean en toques para quemar el "jachi' $i$ " (granos duros) que aparecen en el dorso de la mano. De un brote nuevo que contenga unos doce nudos, puesto al calor, se extrae líquido que se pone en una botella y luego se emplea en masajes contra las hinchazones provocadas por las paperas en el cuello y los testículos. El agua de la maceración de trozos de caña se usa como hemostático en caso de heridas. Las cañas reducidas a cenizas y mezcladas con cera de abejas silvestres se usan para pintarse líneas en la cara, el cuerpo o las extremidades, a fin de evitar los reumatismos.

Cissampelos pareira L. (Menispermaceae), karugua poã, ychypo mirombre. La decocción bebida es considerada como remedio contra el reumatismo. Los tallitos en el agua para el mate se emplean contra la tos y el dolor de cabeza.

Cissus sp. (Vitaceae), ryguachu ka'a.

Citronella gongonha (Mart.) R.A. Howard (Icacinaceae), ka'a rã. Emplean las hojas como sucedáneo de la yerba mate.

Citrullus lanatus (Thunb.) Matsum. \& Nakai ssp. vulgaris (Schrod. ex Eckl. \& Zeyh) Fursa (Cucurbitaceae), chandia, chanjáu. La decocción de las semillas mezclada con Cynodon dactylon, se toma para el dolor de cabeza.

Citrus x aurantium L. (Rutaceae), naranka ka'aguy. El té de las hojas se bebe contra la tos.

Citrus sp. (Rutaceae), naranka'y.

Clematis bonaerensis Juss. ex DC. (Ranunculaceae), koto. Los estigmas quemados se colocan sobre las heridas para detener la sangre.

Clematis montevidensis Spreng. var. montevidensis (Ranunculaceae), koto. Mismo uso que la anterior.

Cnidoscolus albomaculatus (Pax) I.M. Johnst. (Euphorbiaceae), pynõ guachu, pynõ. La raíz en decocción caliente se recomienda en casos de reumatismo, a razón de tres cucharadas mañana y tarde.

Coix lacryma-jobi L. (Poaceae), kapi’i a. Lo cultivan, emplean los granos para cuentas de collares y, pisados en agua fría o tibia, como bebida contra los vómitos.

Coleatania prionitis (Nees) Soreng (Poaceae), kapi'i pororo. Se usa para techar.

Collaea stenophylla (Hook. \& Arn. ) Benth. (Fabaceae), pyky ka'a. Mismo uso que Buchnera longifolia.

Combretum fruticosum (Loefl.) Stuntz (Combretaceae), ychypo pytã, ychypo ratã, aña kygua. La hoja en el tereré se emplea como refrescante, y en decocción como vermicida.

Commelina erecta L.var. erecta (Commelinaceae), karaja ka'a, kochi ka'a. El agua que se junta en las brácteas de la flor, se vierte sobre los ojos en caso de conjuntivitis. La decocción de la planta se usa en lavados de cabeza para calmar ataques de locura.

Convolvulus sp. (Convolvulaceae), ychypo mbói.

Conyza bonariensis (L.) Cronquist (Asteraceae), mbu'y. La decocción de las raíces se usa en buches para calmar dolores de diente.

Copaifera langsdorfii Desf. (Fabaceae), kupa'y. La corteza mezclada con corteza de Parapiptadenia rigida se usa para curtir cueros. Para ello, se ponen trozadas en un recipiente con agua y se introduce el cuero durante 8 a 10 días.

Copernicia alba Morong (Arecaceae), karanda.

Cordia americana (L) Gottschling \& J. S. Mill. (Boraginaceae), guajuvyra, guajayvi. La corteza se pone en el mate y se bebe para calmar la tos. La decocción de la misma se da a las mujeres para cortar la pérdida de sangre luego del parto. La decocción de las hojas se bebe contra la tos y la gripe, la madera del cerne se utilizan para 
hacer arcos y puntas de flecha.

Cordia trichotoma (Vell.) Arráb. ex Steud (Boraginaceae), apyteryvi. La decocción de la corteza se toma contra la acidez del estómago.

Cordia sp. (Boraginaceae), gajo ka'a ka'aguy.

Cordyline spectabilis Kunth \& Bouché (Agavaceae), u'yvarã. La decocción de los brotes y yemas terminales se emplea como diurético para los ancianos, la misma mezclada con corteza de Jacaranda micrantha se usa como bebida contra las purgaciones. Para curar aires se mezclan los brotes en agua fría y se dan masajes en el líquido. La decocción se toma también contra la tos (u'u), y la de los tallos tiernos para el dolor de vientre. La planta se usa para techar, y las hojas, para ataduras de poca duración.

Craniolaria argentina Speg. (Martyniaceae), urupe, jatevu ka'a ñu. El té de las hojas se aconseja para calmar los dolores del parto.

Croton missionum Croizat (Euphorbiaceae), jate'i ka'a.

Croton urucurana Baill. (Euphorbiaceae), moã katĩ. La decocción de brotes e inflorescencias se toma en caso de dolores de vientre.

Croton sp. (Euphorbiaceae), ñerumi para.

Cucumis melo L. (Cucurbitaceae), merõ.

Cucurbita maxima Duch. (Cucurbitaceae), kuara pepẽ. La semilla pisada junto con la raíz de kapi'i pẽ (Paspalum notatum) y semillas de sandía machacada, sirve para preparar un té que se usa como remedio fresco.

Cucurbita moschata Duch. (Cucurbitaceae), andai.

Cuphea glutinosa Cham. \& Schltdl. (Lythraceae), ychypo perẽ. Los gargarismos hechos con la decocción de la planta se usan contra inflamaciones de garganta.

Cuphea linarioides Cham. \& Schltdl. (Lythraceae), mbói ka'a.

Cybistaxantisyphilitica(Mart.)Mart.(Bignoniaceae), tajy chĩ. tajy morochĩ. Para no tener más hijos, el marido y la mujer beben oportunamente la decocción de la corteza.

Cyclanthera hystrix (Gillies ex Hook. \& Arn.) Arn. (Cucurbitaceae), kravachita. Comen las frutas crudas.

Cynodon dactylon L. (Poaceae), kapi’i poñy, kapi'i pẽ. La decocción de la raíz es considerada como remedio fresco y, mezclada con semilla de sandía, se toma como remedio para el dolor de cabeza. Se pone la planta cubriendo el piso de los ranchos, para refrescar y como cama.
Cyperus entrerianus Boeck. (Cyperaceae), piri. Con los tallos aplastados se trenzan sogas.

Cyperus sp. (Cyperaceae), kapi’i aimbe, aiño.

Cyperus sp. (Cyperaceae), kapi'i piri.

Cyperus sp. (Cyperaceae), piri. Para hacer salir la larva de la ura basta con hacer un nudo flojo con la hoja en el aire y tirar de golpe de los extremos hasta que se ajuste.

Cyperus sp. (Cyperaceae), piri'i.

Dalbergia frutescens (Vell.) Britton (Fabaceae), jyva'y, jyva rachy ja, yvyra ponge, ychapy'y, chapy'y. La decocción de la corteza se usa para el lavado de aftas, llagas bucales y para combatir las queresas de la boca de los niños (miasis), y por la mañana temprano, para el lavar la cabeza.

Desmanthus paspalaceus (Lindm.) Burkart (Fabaceae), guachu kumanda, tamonge pytã'i.

Desmanthus tatuhyensis Hoehne (Fabaceae), guachu kumanda, tamonge pytã'i.

Desmodium affine Schltdl. (Fabaceae), guachu ka'a.

Desmodium incanum DC. (Fabaceae), taa-taa. Para eliminar las queresas de la boca se lavan con la decocción de la raíz con sal.

Diatenopteryx sorbifolia Radlk. (Sapindaceae), yvyra porã. Para cortar la sangre de las heridas se emplea la corteza en decocción, sola o mezclada con trozos de tallo de Paullinia pinnata.

Dicella nucifera Chodat (Malpighiaceae), guajaki manduvi, ychypo ava. Consumen las frutas, para lo cual la asan con cáscara y luego la pelan.

Dichorisandra hexandra (Aubl.) Standl. (Commelinaceae), tupãchy recha'i, jachyta ka'a, karaja ka'a. Para la conjuntivitis, se lavan los ojos por la mañana durante varios días con la decocción de la planta. Con las hojas machacadas con agua fría lavan la cabeza de los niños para que estos no contraigan enfermedades. La plantan en los patios, de adorno.

Digitaria sanguinensis (L.) Scop. (Poaceae), kapi'i.

Digitaria sp. (Poaceae), kapi'i pororo.

Dioscorea sp. (Dioscoreaceae), guachu kara.

Dioscorea sp. (Dioscoreaceae), inambu jety. Comen los tubérculos, crudos, cocidos o tostados.

Dioscorea sp. (Dioscoreaceae), karachĩ.

Dorypteris concolor (Langsd. \& Fisch.) Kuhn (Pteridaceae), kurandyjo guachu, amambái. Las hojas se agregan al agua del mate, contra el dolor de cabeza. Los viejos la emplean de la misma manera para evitar enfermedades. 
Drymaria cordata (L.) Willd. ex Roem. \& Schult. (Caryophyllaceae), yvoty.

Eichhornia crassipes (Mart.) Solms (Pontederiaceae), aguape purũ'a. Las partes hinchadas se cocinan y se bebe el líquido, solo o en el mate, en caso de enfermedades del riñón.

Elyonurus sp. (Poaceae), kapi'i, kapi'i ravyju.

Enterolobium contortisiliquum (Vell.) Morong (Fabaceae), chimbo'y, chimbo morochĩ. La decocción de la corteza se usa en lavados de cabeza para eliminar el cansancio físico en las marchas y en caso de epilepsia (yna kañy), en baños de cuerpo y cabeza. La madera se utiliza para horcones. La corteza socada y colocada en una bolsa se introduce en el agua para embarbascar peces.

Eragrostis neesi Trin. (Poaceae), kapi'i.

Erianthus trinii (Hack.) Hack. (Poaceae), kapi'i guachu, kapi'i pororo. Se usa para techar.

Eryngyum eburneum Decne. (Apiaceae), karaguata po'i.

Eryngyum horridum Malme (Apiaceae), karaguata'i. La raíz en decocción se toma en el agua del mate para el dolor del corazón.

Eryngyum pristis Cham. \& Schltdl. (Apiaceae), karaguata'i. La raíz en decocción se toma en el agua del mate como estomacal.

Eringium sp. (Apiaceae), karaguata.

Erythrina crista-galli L. (Fabaceae), yvyra jakare.

Eucalyptus sp. (Myrtaceae), okarito, karito. El té de las hojas se bebe contra la tos y la gripe. Se las pone en el agua del mate para el mismo fin.

Eugenia involucrata DC. (Myrtaceae), yvyra jepiro. Comen las frutas.

Eugenia lilloana D. Legrand (Myrtaceae). Comen las frutas.

Eugenia pyriformis Cambess. var. pyriformis (Myrtaceae), guavira mirĩ, yvaei mirĩ. Comen las frutas.

Eugenia pyriformis Cambess. var. uvalha (Cambess.) D. Legrand (Myrtaceae), ñangapiri mirĩ. Comen las frutas.

Eugenia uniflora L. (Myrtaceae), pitanga, añangapiri. La decocción de las hojas se bebe como estomacal. Comen las frutas.

Eupatorium verbenaceum DC. (Asteraceae), teju'i ka'a.

Eupatorium sp. (Asteraceae), kavara ka'a. Las hojas se ponen en el mate para calmar el dolor de barriga.

Eupatorium sp. (Asteraceae), ñerumi. La decocción se usa en el lavado de heridas.

Eupatorium sp. (Asteraceae), pira ka'a.

Euphorbia papillosa A. St.-Hil. (Euphorbiaceae), pira ka'a. Se frota la línea de pescar con la planta para atraer peces.

Euphorbia serpens Kunth (Euphorbiaceae), jatevu ka'a, tupãchy kamby. La decocción se toma como bebida, para curar la aftosa y las enfermedades del hígado.

Euphorbia sp. (Euphorbiaceae). tupãchy kamby. Se ponen las hojas en el mate como refrescante.

Euterpe edulis Mart. (Arecaceae), jejy.

Evolvulus sericeus Sw. (Convolvulaceae), anguja ka'a. Se machaca la planta con un trozo de labio de rata de monte y se pone la mezcla en un trapito, con este se frotan las cimbras y las trampas para atraer a dicho animal.

Familia indet. (Pteridophyta), amambái. Decocción estomacal.

Familia indet. (Pteridophyta), amambái. Las hojas en el agua para el mate se emplean contra el dolor de garganta y tos.

Familia indet., aguara yva'i. El jugo de las hojas se hecha sobre los ojos para curar la conjuntivitis.

Familia indet., ychypo. Tallos para manija de cestos.

Ficus sp. (Moraceae), kava chingy, ũapo'y. Una gota de látex se coloca sobre la piel, en el lugar atacado, para extraer la úras. La cáscara se pone en la garganta y se sujetan con trapos para curar el bocio y las paperas.

Forsteronia sp. (Moraceae), ychypo kamby. El látex, en aplicaciones, se recomienda contra el nacido (granos producidos a causa de una infección).

Froelichia aff. paraguayensis Chodat (Amaranthaceae), torypa.

Galactia benthamiana Michelli (Fabaceae), aguara po.

Galactia boavista (Vell.) Burkart (Fabaceae), aguara po.

Galactia striata (Jacq.) Urb. var. striata (Fabaceae), guachu kumanda.

Galphimia brasiliensis (L.) A. Juss (Malpighiaceae), churuku'a ka'a. Para calmar el dolor de oído, se introduce en este las hojas tostadas y mojadas en agua fría, tapando luego con un trozo de algodón.

Género indet. (Bignoniaceae), ychypo.

Género Indet. (Bignoniaceae), ychypo. Usan los tallos para hacer canastos.

Glandularia peruviana (L.) Small (Verbenaceae), yvoty, pironko ka'a. 
Glandularia sp. (Verbenaceae), jagua ka'a.

Gleditsia amorphoides (Griseb.) Taub. (Fabaceae), mbogua ju.

Glycine max (L.) Merr. (Fabaceae), kumanda choka. Conocen esta especie pero rara vez la cultivan.

Gomidesia palustris (DC.) Kausel (Myrtaceae), typycha'y. Comen las frutas, beben la decocción de la corteza contra el dolor del estómago.

Gomphrena celosioides Mart. (Amaranthaceae), perurija . La maceración de la planta en agua se emplea para el lavado de ojos en caso de conjuntivitis, ver Allophylus edulis.

Gossypium herbaceum L. (Malvaceae), mandyju.

Guadua chacoensis (Rojas Acosta) Londoño \& P.M.Peterson (Poaceae), takuaruchu. Con la corteza cortada en tiras hacen canastos.

Guadua trinii (Nees) Nees ex Rupr. (Poaceae), pekuru. Con la corteza cortada en tiras hacen canastos.

Guarea guidonia (L.) Sleumer (Meliaceae), chegrijo. La corteza en decocción sirve para lavados y como bebida para curar heridas; en el mate, contra dolores internos.

Gynerium sagittatum (Aubl.) P. Beauv (Poaceae), takuaty. Mismos usos que Arundo donax.

Handroanthus albus (Cham.) Mattos (Bignoniaceae), tajy morochĩ.

Handroanthus heptaphyllus (Vell.) Mattos (Bignoniaceae), tajy, tajy ũ. tajy ovy. Ver Blechnum australe.

Hedychium coronarium J. K König. (Zingiberaceae), pegua'õ. Los rizomas se ponen en el agua para el mate en caso de dolor de vientre.

Helietta apiculata Benth. (Rutaceae), yvyra ovy. Para curar el dolor de cabeza, se debe lavan la cabeza con agua que contenga hojas machacadas.

Heliocarpus popayanensis Kunth (Tiliaceae), yvyra pire ũ.

Heliotropium phylicoides Cham. (Boraginaceae), eira ka'a. En la decocción, se bañan a los perros para curarles enfermedades internas.

Heliotropium sp. (Boraginaceae), jachyta ka'a.

Hennecartia omphalandra (Monimiaceae), yvyra karai. Se toma el agua de decocción de las hojas en el mate cuando se levantan mareados.

Hexachlamys edulis (O. Berg) Kausel \& D. Legrand (Myrtaceae), yvaei. Comen las frutas, la decocción de la corteza y a razón de un litro cada hora se bebe para el corazón.

Hippeastrum sp. (Amarylidaceae), kapi'i pẽ.

Holocalyx balansae Micheli (Fabaceae), yvyra pepẽ.
Con el cerne hacen puntas de flecha para cazar. Hybanthus parviflorus (Mutis ex L.f.) Baill. (Violaceae), aguara'i ka'a.

Hydrocotyle bonariensis Lam. (Apiaceae), aguara namby'i. Contra los dolores se dan friegas con las hojas machacadas con grasa de zorro.

Hydrocotyle exigua (Urb.) Malme (Apiaceae), jachyta ka'a, jachyta, uru ka'a. Toman las hojas en el mate como refrescante.

Hydrocotyle leucocephala Cham. \& Schltdl. (Apiaceae), jachyta ka'a, jachyta yrupe. La decocción se emplea en el lavado de los ojos en caso de conjuntivitis.

Hypochaeris chillensis (Kunth) Hieron. (Asteraceae), chikoria mirĩ. Comen las hojas crudas en ensalada $\mathrm{y}$ cocidas como verdura.

Hypochaeris microcephala (Sch. Bip.) Cabrera var. albiflora (Kuntze) Cabrera (Asteraceae). chikoria mirĩ. Comen las hojas crudas en ensalada y cocidas como verdura.

Hyptis althaeifolia Pohl ex Benth. (Lamiaceae), chirka. Las mujeres restriegan las hojas en las manos hasta que suelten un líquido amarillento rojizo con el cual se colorean y perfuman las mejillas.

Hyptis floribunda (Briq.) Briq. Ex Micheli (Lamiaceae), kangue'i, poréo'i. El té se usa para calmar el dolor de vientre de las mujeres.

Hyptis mutabilis (Rich.) Briq. (Lamiaceae), tapẽ kue, taguato ka'a. Para el dolor de la cintura, se dan friegas al acostarse con la decocción de los ápices floríferos.

Ibicella lutea (Lindl.) Van Eselt. (Martyniaceae), mba'e pochy rova, jatevu ka'a.

Ilex dumosa Reissek (Aquifoliaceae), ka'a yna, ka'a chiri. Usan las hojas como sucedáneo de la yerba mate.

Ilex paraguarienses A. St.-Hil. (Aquifoliaceae), ka'a. Usan mucho como bebida en mate o en tereré. Seca y molida, se aplica sobre las heridas como hemostático. Las hojas verdes atadas a la cabeza con trapo son usadas contra las cefalalgias.

Inga marginata Willd. (Fabaceae), inga'y, inga. Comen la parte interna de los frutos.

Inga verna Willd. subsp. affinis (DC.) T.D. Penn. (Fabaceae), inga. Comen la parte interna de los frutos.

Ipomoea batatas (L.) Lam. (Convolvulaceae), jety. Ipomoea descolei O’Donell (Convolvulaceae), kata. Ipomoea sp. (Convolvulaceae), jaku pycha. 
Ipomoea sp. (Convolvulaceae), jety rã.

Ipomoea sp. (Convolvulaceae), ychypo ñu.

Ipomoea sp. (Convolvulaceae), ypoty.

Iresine diffusa Humb. \& Bonpl. ex Willd. (Amaranthaceae), kangue'i, tapẽ kue. La decocción de la planta se bebe en caso de recalcaduras y golpes.

Isostigma peucedanifolium (Spreng.) Less. (Asteraceae), yvoty ãiño.

Jacaranda micrantha Cham. (Bignoniaceae), karova, yvyra moã. La decocción de la corteza se bebe en caso de purgaciones, sola o mezclada con brotes de Cordyline spectabilis.

Jacaranda puberula Cham. (Bignoniaceae), para para'y, pra para'y. La decocción de sus hojas o de la corteza es bebida por las mujeres después del parto.

Jacaratia spinosa (Aubl.) A. DC. (Caricaceae), jarakachĩ’a guachu. Comen las frutas cocidas o asadas al rescoldo.

Jacquemontia blancheti Moric. (Convolvulaceae), jety rã.

Jacquemontia sp. (Convolvulaceae), inambu ychypo.

Justicia sp. (Acanthaceae), kangue'i, maino rembi'u, karaja ka'a, maino ka'a. La planta, puesta en agua, se recomienda para curar el vicio de la embriaguez.

Kyllinga odorata Vahl. (Cyperaceae), kapi’i kachĩ. Contra los vermes intestinales se toma la decocción de las raíces.

Laciasis sp. (Poaceae), takuary.

Lagenaria siceraria (Molina) Standl. (Cucurbitaceae), i'a rembo. Para mate (recipiente).

Leonurus sibiricus L. (Lamiaceae), mbu'y. La decocción de la raíz se usa como desinfectante para el lavado de heridas.

Leptochloa virgata (L.) P. Beauv. (Poaceae), kapi'i.

Leuhea divaricata Mart. (Tiliaceae), ychongy. La decocción de la corteza ubicada del lado que sale el sol, se emplea contra la tos, sola o mezclada con corteza de Cordia americana. También para la afonía o ronquera, debiendo tomarse a razón de un litro por hora. En buches y bebidas se recomienda contra las queresas de la garganta y aftas bucales.

Lippia lippioides (Cham.) Rusby (Verbenaceae), jagua ka'a. Comen las frutas.

Lippia lupulina Cham. (Verbenaceae), uru ka'a.
Las hojas se ponen en el mate para combatir la fiebre en los hombres.

Lippia salviaefolia Cham. (Verbenaceae), niño rupa.

Lonchocarpus muehlbergianus Hassl. (Fabaceae), yvyra kachĩ, inga guachu, guaja chimbo. Contra los vermes intestinales beben la decocción de la corteza, brotes u hojas. La corteza se usa para embarbascar peces.

Lonchocarpus campestris Mart. Ex Benth. (Fabaceae), yvyra kachĩ. La decocción de las hojas, cogollos o corteza, se toma a razón de cuatro o cinco cucharadas contra las lombrices intestinales y dolores de vientre; la maceración de la corteza en agua fría como estomacal.

Lophophytum leandri Eichler (Balanophoraceae), ita poty. Se ponen trozos de la inflorescencia o el rizoma en el mate, y se da a los moribundos para que revivan.

Ludwigia elegans (Cambess.) H. Hara(Onagraceae), ñerumi mirĩ. Las hojas secas y reducidas a polvo se aplican sobre las heridas.

Luffa cilíndrica M. Roem. (Cucurbitaceae), kuaray'a. La fruta se llama y'a kue.

Macfadyena unguis-cati (L.) A. Gentry (Bignoniaceae), chivi ka'a, yvy'a, jagua pinda. La decocción de la raíz, o su maceración en agua fría se toma contra las mordeduras de víboras, y hervida, como galactógeno. Con los tallos se hacen ataduras en las piernas para que el veneno no suba en caso de mordeduras de víboras.

Maclura tinctoria (L.) Steud. (Moraceae), tata yva, tata jyva. Comen las frutas. Con la decocción concentrada de la corteza pintan los cueros de rojo. La decocción del cerne sirve para curtir cueros a los que da color amarillo, lo mismo que los géneros y la ropa.

Malvastrum coromandelianum (L.) (Malvaceae), typycha ũ.

Manettia inflata Sprague (Rubiaceae), maino ka'a.

Manihot esculenta Crantz (Euphorbiaceae), mandy'o, manjy'o.

Manihot grahamii Hook. (Euphorbiaceae), mandy'o, manjy'o, guachu manjy'o. Aseguran que su follaje es tóxico para el ganado. La maceración de las hojas en agua fría se usa en lavados de cabeza para refrescarse.

Maranta sobolifera L. Andersson (Marantaceae), pegua'õ, tarope. Con una planta machacada en agua fría, se lavan la cabeza en caso de 
insolación; la decocción de las frutas es tomada por las mujeres para evitar embarazos; las hojas cocinadas en agua se adhieren a la zona hepática mediante grasa de pato, cuando duele el hígado.

Marchantia sp. (Marchantiaceae), karumbe ka'a.

Marchantia sp. (Marchantiaceae), yga'u.

Maytenus ilicifolia Mart. ex Reissek (Celastraceae), kangorocha, yvyra rapõ ju. La corteza en el agua para el mate es considerada como una panacea contra todas la enfermedadesy, en especial, en casos de dolores de vientre; ver Setaria poiretana.

Melia azedarach L. (Meliaceae), paraicho. Con la decocción de las hojas se dan masajes en casos de calambres musculares.

Melicoccus lepidopetalus Radlk. (Sapindaceae), yva poõ. Comen la fruta; en Misiones es cultivada.

Melochia ulmarioides A. St. -Hil. (Sterculiaceae), ñandu ka'a. Para las mordeduras de arañas se frotan con las hojas, luego lavan y tapan con un trapo atado.

Melothria cucumis Vell. (Cucurbitaceae), anguja chanjáu, apere'a chanjáu. Comen las frutas.

Melothria pendula L. (Cucurbitaceae), anguja chanjáu.

Melothria warmingii Cogn. (Cucurbitaceae), anguja chanjáu. Comen las frutas.

Mentha rotundifolia (L.) Huds. (Lamiaceae), ka'a porã. Ver Setaria sulcata.

Merostachys claussenii Munro (Poaceae), takua, takua'y, takuapi. Con un trozo de caña entre dos nudos fabrican una clase de flauta que llaman "mimby"; usan las cañas para armazón de cestos.

Miconia tristis Spring (Melastomataceae), ka'a yta. La decocción de las ramitas, cortadas en luna nueva, se emplea para el lavado de dientes de los niños con el fin de que estos duren siempre sanos.

Microgramma vacciniifolia ( Langsd. \& Fisch ) Copel.(Polypodiaceae), ambere ka'a, ambere mby. Se emplea en el agua del mate para el mal de riñones; mezclado con doradilla, la beben las mujeres contra el pasmo y se recomienda como remedio caliente; las hojas enrolladas se introducen en las orejas, para curar sorderas repentinas.

Mikania glomerata Spreng. (Asteraceae), ychypo’y, katu ka'a, ychypo rõ, ka'a ro. La decocción de la plata, en baños, se usa contra la fiebre.

Mimosa cruenata Benth. (Fabaceae), tamonge guachu.

Mimosa obstrigosa Burkart (Fabaceae), tamonge guachu.

Mimosa paupera Benth. (Fabaceae), tamonge'i.

Mimosa polycarpa Kunt (Fabaceae), tamonge guachu e'ỹ.

Mimosa selloi (Benth.) Benth. (Fabaceae), tamonge'i.

Mimosa uliginosa Chodat \& Hassl. (Fabaceae), tamonge guachu.

Mimosa velloziana Mart. (Fabaceae), tamonge guachu.

Musa sp. (Musaceae), pakova guachu, pakova de oro, pakova karapẽ.

Myrceugenia euosoma (O. Berg) D. Legrand (Myrtaceae), jaku rembi'u. Comen las frutas.

Myrcia anomala Cambess. (Myrtaceae), guavira mirĩ. Comen las frutas.

Myrcia bombycina (O. Berg) Kiaersk. (Myrtaceae), typycha ũ. Comen las frutas.

Myrcianthes pungens (O. Berg) D. Legrand (Myrtaceae), yva viju, guavira mirĩ. Emplean la decocción de la corteza, a razón de un litro diario, como febrífugo. Comen las frutas.

Myrocarpus frondosus Allemão (Fabaceae), yvyra paje. La decocción de la corteza se toma contra el dolor de vientre, y se usa en lavados para curar heridas; la decocción de la corteza y hojas se toma contre cefalalgias.

Myrsine párvula (Mez) Otegui (Myrsinaceae), pira rembi'u.

Myrsine sp. (Myrsinaceae), apere'a ka'a. Con la madera hacen puntas de flecha. La decocción de la corteza se emplea contra el dolor de vientre. La corteza se frota contra las cuerdas del violín para mejorar su sonido.

Nectandramegapotamica(Spreng.) Mez(Lauraceae), aju'y, aju'y morochĩ, aju'y ũ. La decocción de las hojas se recomienda como vermífugo.

Nicotiana tabacum L. (Solanaceae), petỹ. El agua de la decocción de las hojas se usa para el lavado de heridas, ver Sapium.

Noticastrum macrocephalum (Baker) Cuatrec. (Asteraceae), petỹ ra mirĩ.

Ocimun nudicaule Benth. (Lamiaceae), ka'a rupe, jayrogue rupe. La raíz se pone en el agua del mate para mejorar su sabor. Seca y rallada, se mezcla con el tabaco de cigarrillo en casos de asma. 
Ocotea diospyrifolia (Meisn.) Mez (Lauraceae), aju'y ju, aju'y ũ.

Ocotea puberula (Rich.) Nees (Lauraceae), aju'y guaika, aju'y ũ. La madera sirve para hacer horcones.

Olyra latifolia L. (Poaceae), takuarembo'i.

Oncidium biflorum Barb. Rodr. (Orchidaceae), mbaraka poã. Se calienta una pelotita con un poco de agua, y la cola que suelta se emplea para encolar guitarras.

Osmunda regalis L. var. spectabilis (Willd.) A.Gray (Osmundaceae), ñachĩ’u rã. La decocción de las plantas se usa para el lavado de los ojos por las mañanas, y tomada en el mate contra los dolores de garganta.

Oxalis conorrhiza Jacq. (Oxalidaceae), inambu jety. La decocción de la planta en medio litro de agua se da a tomar a los niños que orinan poco.

Oxalis sp. (Oxalidaceae), kavure'i ka'a. Se conservan las hojas, junto con plumas de kavure, atadas en un trapito; cuando se quiere enamorar a una mujer se le comunica que uno ha preparado dicho kurundu, con ese fin.

Oxypetalum brachystemma Malme (Asclepiadaceae), mbóichy ka'a. Dicen que la víbora mbóichy come las hojas para despertar su apetito. El látex se aplica sobre la mordedura de este reptil y luego se cubre con la hoja y se ata con un trapo.

Oxypetalum sp. (Asclepiadaceae), ychypo.

Oxypetalum sp. (Asclepiadaceae), yryvu ka'a.

Panicum repens L. (Poaceae), kapi'i pẽ.

Panicum sp. (Poaceae), kapi'i.

Panicum sp. (Poaceae), takuarembo'i.

Paramyrciaria delicatula(DC.) Kausel(Myrtaceae), pitanga mirĩ. Comen las frutas. La decocción de la corteza se toma para el dolor de estómago.

Parapiptadenia rigida (Benth.) Brenan (Fabaceae), kurupa'y, kurupa'y morochĩ. La decocción de la corteza se bebe contra la tos y la gripe, y se emplea para el lavado de heridas. Se usa en curtiembre, ver kupa'y.

Paspalum conjugatum P.J. Bergius (Poaceae), kapi'i pẽ.

Paspalum coryphaeum Trin. (Poaceae), kapi'i, kapi'i guachu. Se usa para techar.

Paspalum nicorae Parodi (Poaceae), kapi'i.

Paspalum notatum Flüggé (Poaceae), kapi’i pẽ, kapi'i poñy. Los rizomas machacados se ponen en el agua del mate, en caso de dolor de riñones; ver Cucurbita maxima como remedio fresco.
Paspalum plicatulum Michx. (Poaceae), kapi'i.

Paspalum urvillei Steud. (Poaceae), kapi'i.

Paspalum sp. (Poaceae), kapi'i poñy. La decocción de la planta se emplea como abortivo y anticonceptivo.

Passiflora sp. (Passifloraceae), guachu ka'a. Comen las frutas.

Passiflora sp. (Passifloraceae), mburukuja mirĩ. Comen las frutas.

Passiflora sp. (Passifloraceae), mburukuja. Comen las frutas.

Passiflora sp. (Passifloraceae), mburukuja'i. Comen las frutas.

Paullinia elegans Cambess. (Sapindaceae), ychypo ũ.

Paullinia sp. (Sapindaceae), ychypo timbo, chimbo. Las hojas y la corteza en trozos de medio metro, machacadas con un pal, o se emplean para embarbascar peces; para ello se colocan trozos en unas bolsa que se introduce en el agua; los peces tardan de media a una hora en morir, ver Diatenopteryx sorbifolia.

Pavonia subrotunda A. St. -Hil. \& Naudin (Malvaceae), maino ka'a. Las hojas se toman en el mate contra la fiebre.

Peltophorum dubium (Spreng.) Taub. (Fabaceae), yvyra pytã. La decocción de la corteza en lavados se usa para eliminar las queresas de la boca.

Peperomia sp. (Piperaceae), Churukua ka'a ka'aguy. Se bebe la decocción como remedio fresco.

Petiveria alliacea L. (Phytolacaceae), pipi. La decocción de la planta es considerada febrífuga; la de la raíz se emplea como bebida contra la tos y la gripe. Con la decocción de la planta, junto con cenizas, se dan masajes en las piernas para activar la circulación de la sangre. La planta molida se esparce sobre los almácigos de tabaco para evitar el ataque de gusanos.

Pfaffia tuberosa (Spreng.) Hicken (Amaranthaceae), perurija. Se emplean del mismo modo que Gomphrena celosioides.

Pharus lappulaceus Aubl. (Poaceae), ka'i aro. El grano molido o la hoja machacada y diluida en leche de mujer, se emplea en gotas, cada dos o tres horas, para curar la conjuntivitis.

Phaseolus clitorioides Mart. ex Benth. (Fabaceae), popo ka'a.

Phaseolus vulgaris L. (Fabaceae), kumanda.

Philodendron bipinnatifidum Schott (Araceae), guembe. Comen las frutas. Cortan en tiras la 
corteza de la raíz "guembepi" y con ellas forran los arcos y atan las manijas y boca de canastos y cestas.

Phyllanthus sellowianus (Klotzsch) Müll. Arg. (Euphorbiaceae), charandy morochĩ.

Phyllantus sp. (Euphorbiaceae), ychau ka'a.

Physalis viscosa L. (Solanaceae), kaite mirĩ. Comen las frutas.

Phytolacca dioica L. (Phytolaccaceae), umby, yvyra guachu, yvyra yvypy guachu. Baños con la maceración o decocción de las hojas se usan para hacer engordar a los niños flacos.

Picrasma crenata (Vell.) Engl. (Simaroubaceae), yvyra pire chĩ, yvyra pire morochĩ. La corteza en decocción se bebe contra cefalalgias y dolores musculares.

Pilocarpus pennatifolius Lem. (Rutaceae), yvyra petái. Con la lámina del cuchillo se raspa la corteza; la raspadura se mezcla con grasa de gallina, y con esta pasta se obturan las caries dentales para calmar el dolor. Con la madera se hacen puntas de flechas.

Piper sp. (Piperaceae), jai rogue.

Piper sp. (Piperaceae), pariparova, jaguarundy, jaguarundy guachu, tuja renapy'a, pariparova guachu. Usan la infusión de las hojas para calmar el dolor de vientre, fiebre, tos y gripe de las criaturas; la decocción de las hojas es empleada contra chevo'i (parásitos intestinales).

Piptocarpha sellowi (Sch. Bip.) Baker (Asteraceea), ka'a mbara. La decocción, en el mate, se toma contra el dolor de cabeza.

Pistia stratiotes L. (Araceae), paa guata, akarachĩ vai poã. Las plantitas jóvenes se ponen en el agua del mate contra las cefalalgias, o se toman en agua fría.

Plinia rivularis (Cambess.) Rotman (Myrtaceae), yvaporãity. Comen las frutas. Con el palo y la corteza preparan una decocción que beben contra el dolor de estómago; la corteza se toma contra el sarampión. Con las ramas atadas con guembepi fabrican trampas para cazar venados.

Plinia trunciflora (O. Berg) Kausel (Myrtaceae), yva purũ. Comen las frutas.

Poikilacanthus glandulosus (Nees) Ariza (Acanthaceae), kangue'i, tujarenapy'a. Las hojas en el mate, se ingieren como refrescante.

Poiretia tetraphylla (Poir.) Burkart (Fabaceae), ychau ka'a, kavara ka'a. La decocción de la planta se emplea contra las enfermedades internas. Ponen las hojas en el mate para darle mejor sabor.

Polygonum punctatum Elliott (Polygonaceae), ka'a tái. Algunos dicen que se trata de una planta tóxica, otros emplean la decocción de la raíz como bebida para secar el apéndice, y la de la planta, en lavado de heridas, granos que pican hinchazones y fuego de San Antonio.

Polygonum sp. (Polygonaceae), katái. La decocción de las hojas, en baños y en tomas, para dolores del corazón.

Polypodium sp. (Polypodiaceae), amambái, jagua ka'a, mburika ka'a. La decocción se bebe contra el dolor de vientre y para no tener más hijos.

Pontederia rotundifolia L. F. (Pontederiaceae), karumbe ka'a.

Porophyllum ruderale (Jacq.) Cass. (Asteraceae), mbu'ychĩ, mbu'ytĩ, mbu'yrẽ. Contra el dolor de la vista, ponen la planta machacada en agua fría y se lavan los ojos en la mañana temprano, o bien machacan las hojas agregándoles leche de mujer y usan la mezcla en gotas. Los que ven poco, se lavan la cara y los ojos con la decocción de la planta, a fin de mejorar la visión. Dicha decocción, en baños, se aconseja como febrífugo.

Portulaca oleracea L. (Portucaceae), karuru mirĩ. Comen los brotes tiernos en ensalada, y las hojas hervidas a modo de verdura.

Pouteria gardneriana (A. DC.) Radlk. (Sapotaceae), aguai guachu. Comen las frutas cocidas.

Pouteria sp. (Sapotaceae), pakuri vai, aguai chaiju.

Prunus subcoriacea (Chodat \& Hassl.) Koehne (Rosaceae), aju'y vovõ. La decocción de la corteza se usa en baños contra las hinchazones.

Prunus sp. (Rosaceae), yvaro. La maceración de las hojas en agua fría se toma contra la fiebre; la decocción de las hojas se usa en baños y masajes para activar la circulación sanguínea de las piernas, y eliminar el cansancio de grandes caminatas; el agua de maceración de las hojas se recomienda en lavados, en casos de cefalalgias.

Pseudananas sagenarius (Arruda) Camargo (Bromeliaceae), karaguata re'i. Comen las frutas y extraen fibras de los rizomas y hojas.

Pseudoechinolaena polystachya (Kunth) Stapf (Poaceae), kapi'i pororo ka'agüy.

Psidium australe Cambess. (Myrtaceae), aracha mirĩ. Comen las frutas.

Psidium australe var. argenteum (O. Berg) Landrum (Myrtaceae), aracha ñu, guavira mirĩ. Comen 
las frutas, los cogollos macerados en agua se emplean para el lavado de las cabezas de las criaturas con fiebre. La corteza en decocción se toma en caso de dolores intestinales.

Psidiumgrandifolium DC.(Myrtaceae), guavijumirĩ. Comen las frutas que son muy apreciadas.

Psidium guajava L. (Myrtaceae), aracha. Comen las frutas. La decocción de las hojas y la corteza es considerada un eficaz remedio contra los dolores de barriga.

Psidium guineense Sw. (Myrtaceae), aracha. Comen las frutas.

Psidium missionum D. Legrand (Myrtaceae), aracha. Comen las frutas.

Psychotria carthagenensis Jacq. (Rubiaceae), tapichĩ ka'a.

Pteridium arachnoideum (Kaulf.) Maxon (Dennstaedtiaceae), amambaí.

Pterogyne nitens Tul. (Fabaceae), karaja'y, tembetary mirĩ. La decocción caliente se toma contra el catarro bronquial.

Pycnoporus sanguineus (L.) Murrill (Polyporaceae), urupe'i. La decocción caliente la beben las mujeres para evitar los embarazos.

Rheedia brasiliensis (Mart.) Planch. \& Triana (Clussiaceae), pakuri. Comen las frutas, previamente peladas. La decocción de la corteza se bebe en caso de dolores intestinales; la corteza tostada y dejada en maceración en agua fría, es considerada como bebida frebrífuga.

Rhipsalis lumbricoides (Lem.) Lem. ex SalmDick (Cactaceae), ambere mbói, pengue poã. Cuando se fracturan los huesos de la canilla, se machaca la planta y se ata con un trapo alrededor de la parte fracturada; de la misma forma se usa en caso de mordedura de víboras. Comen la frutas.

Rhipsalis sp. (Cactaceae), pengue ja. En caso de huesos sacados o recalacaduras se dan fricciones con la decocción de la planta.

Rhipsalis sp. (Cactaceae), ychypo petái. La decocción se emplea, en bebida y en lavado, en caso de machucones.

Rhynchosia edulis Griseb. (Fabaceae), jagua pycha.

Rhynchosia lineata Benth. (Fabaceae), guachu ka'a.

Richardia brasiliensis Gomes (Rubiaceae), perurija.

Ricinus communis L. (Euphorbiaceae), amba'y'i.
Las hojas maceradas en agua fría se prepara en líquido para el lavado de la cabeza, en casos de cefalalgias.

Rivina humilis L. (Phytolaccaceae), yvyra apytã. Con las frutas, las mujeres preparan colorete para las uñas, labios y mejillas. Para teñirse, hierven largamente y luego se introduce el hilo y se hierve nuevamente hasta que el color rojo quede fijado.

Rollinia emarginata Schltdl. (Annonaceae), aratiku. Comen la fruta. Con la decocción de la corteza seca pulverizada se prepara una bebida para los dolores intestinales.

Rollinia salicifolia Schltdl. (Annonaceae), aratiku guachu. Mismos usos que Rollinia emarginata.

Roupala montana Aubl. var. brasiliensis (Klotzsch) K.S. Edwards (Proteaceae), mborevy ka'a guachu. La decocción de la corteza se bebe a razón de un litro por hora, en los casos de dolores del corazón.

Ruellia sp. (Acanthaceae), jeruti ka'a.

Ruprechtia laxiflora Meisn. (Polygonaceae), yvyra u'u.

Ruprechtia salicifolia (Cham. \& Schltdl.) C.A. Mey. (Polygonaceae), charandi ũ.

Saccharum officinarum L. (Poaceae), takuarẽ'ẽ.

Salvia sp. (Lamiaceae), chingachu ka'a. Se utiliza como remedio fresco, tomándose en el mate.

Sambucus australis Cham \& Schltdl. (Caprifoliaceae), moã pyro'y. La decocción de las hojas se emplea como bebida contra la gripe; en lavados, en caso de sarampión. Comen las frutas y a veces hacen vino con ellas agregándole alcohol.

Sapium glandulosum (L.) Morong (Euphorbiaceae). kurupi ka'i guachu, Para extraer la larva de las úras se aplica en trocito de hoja de tabaco pegado con látex de esta especie y en el lugar de penetración. El látex aplicado sobre muelas y dientes los hace caer.

Sapium haematospermum Müll. Arg. (Euphorbiaceae), kurupy ka'i. El látex se pone sobre las caries, con lo cual el diente cae.

Schefflera morototoni (Aubl.) Maguire, Steyerm. \& Frodin (Araliaceae), amba'y guachu, amba'y ka'aguy, amba'y guachu ka'aguy. Un trozo de corteza introducido en las caries sirve para voltear las muelas. Las hojas se emplean en decocción contra la tos y la gripe, y la decocción de los brotes, en buches, en caso de dolor de muelas. 
Schinus lentiscifolius Marchand (Anacardiaceae), ryguaja rembi'u. La decocción de la corteza de las raíces se usa en lavados para eliminar queresas de la boca.

Schinus molle L. (Anacardiaceae), yva'y, yva mirĩ.

Schinus terebenthifolius Raddi (Anacardiaceae), ryguaja rembi'u, kutolo ka'a, yryvaja'y. La decocción de la raíz se usa en buches para calmar el dolor de dientes. El agua de maceración de las hojas y ramitas sirve para el lavado de cabeza en caso de cefalalgias. La decocción de la corteza y de las hojas, para la fiebre; y, en buches, contra las aftas de los niños.

Schinus weinmannifolius Engl. (Anacardiaceae), ryguaja rembi'u, moje'i. Las hojas se maceran en agua fría y el macerado se usa en baños para disminuir la fiebre de los niños. La decocción de la corteza sirve para eliminar las queresas de la boca de los niños.

Schizachyrium paniculatum (Kunth) Herter (Poaceae), aguara ruguái.

Scoparia dulcis L. (Scrophulariaceae), uru ka'a.

Scoparia hassleriana Chodat (Scrophulariaceae), poréo, tajy'i. Las mujeres beben la decocción de las hojas quemadas, para evitar embarazos y como abortivo.

Sebastiania brasiliensis Spreng. (Euphorbiaceae), tymba ju'y, yvyra pororo, yvyra piriri. El látex se aplica en el lugar en que se introdujo la larva de la ura, para asfixiarla y hacerla salir.

Selaginella sp. (Selaginellaceae), guaimi rague. La decocción se usa para el lavado de heridas.

Selaginella sp. (Selaginellaceae). y'au.

Senecio brasiliensis Spreng. (Asteraceae). mbu'y.

Senecio sp. (Asteraceae), chikoria'y. Se cocina en agua y medio litro de la decocción se bebe diariamente en caso de dolor de hígado, riñón o estomago.

Senecio sp. (Asteraceae), mbu'y.

Senna pilifera (Vogel) H.S. Irwin \& Barnebay var. pilifera (Fabaceae), guachu kumanda.

Setaria sulcata Raddi (Poaceae), pindo rã, kuchuvirando, kapi'i kuchuvirando. La raíz mezclada con la raíz de Cenchrus myosoides y la corteza de cangorosa, se cocina y se conserva el agua en una botella. Esta se bebe para purificar la sangre. La raíz combinada con raíces de yerba buena, karuru y verbena, se toma en decocción contra dolores internos.

Setaria verticillata (L.) P. Beauv. (Poaceae), kapi'i. Setaria sp. (Poaceae), kapi'i.
Sicyos polyacanthus Cogn. (Cucurbitaceae), y'arã. Sida sp. (Malvaceae), anguja ruguái.

Sida rhombifolia L. (Malvaceae), typycha, typycha ũ. Con las plantas atadas a las extremidades de un palo se fabrican escobas. Los pétalos se introducen en las caries dentales para calmar el dolor, o bien se cocina la planta y se hacen buches. Con las hojas se cubren las heridas para que curen rápido. La decocción de la raíz en buches continuados durante cada hora, se emplea para el dolor de muelas.

Sida urens L. (Malvaceae), tapichĩ ka'a. Las hojas se ponen en el agua del mate para el dolor de barriga.

Smilax campestris Griseb. (Smilacaceae), ychypo ju'u, akarachy poã. La decocción de la raíz en el mate se toma para el dolor de cabeza. La decocción de las frutas, mezclada con corteza de Trichillia catigua, suministra una tintura color gris plomo azulado, que emplean para pintar el Takuapy con el que hacen cestas.

Solanum aff. chenopodioides Lam. (Solanaceae), guachú ky’yĩ, ka'a ite. Opinan que las frutas son venenosas. Las frutas seccionadas se aplican sobre las heridas grandes, mediante un trapo, para curarlas.

Solanum aff. insidiosum Mart. (Solanaceae), ju'u,tuti'a, ju'a para. Las frutas asadas se usan en aplicaciones para madurar forúnculos. La decocción, en baños o fricciones, sirve para disminuir las hinchazones del cuerpo.

Solanum bonariense L. (Solanaceae), guachu,ky’yĩ, ju'a.

Solanum licopersicum Lam. (Solanaceae), juya'a.

Solanum mauritianum Scop. (Solanaceae), ka'õvechingy. La decocción de la raíz, junto con la corteza, se usa en lavados de la boca en casos de dolores dentales.

Solanum pseudocapsicum L. (Solanaceae), ju'a'i. Las frutas seccionadas se aplican sobre las heridas para su curación.

Solanum sisymbriifolium Lam. (Solanaceae), tuti'a, ju'a pytã, ñuati pytã. Comen las frutas, beben el té o la decocción de las raíces en caso de enfermedades del riñón y vejiga; con la decocción se hacen buches para calmar el dolor de muelas. Las frutas reccionadas se aplican sobre heridas para su curación.

Solanum sp. (Solanaceae), guachu ka'a. Los brotes se ponen en el agua del mate para calmar los dolores corporales. 
Solanum sp. (Solanaceae), kavachyngy'i.

Solanum sp. (Solanaceae), ñu'aty, ju'aty.

Solanum sp. (Solanaceae), paroma ka'a.

Sorocea bonplandii (Baill.) W.C.Burger, Lanj. \& Wess.Boer (Moraceae), ñandipa, ñandyta, yvyra karai. Comen las frutas cocidas. Emplean la madera para fabricar manijas y aros de cestos y canastos. El látex se emplea a modo de crema facial, para curar los granos de la cara. Se colocan las hojas en el agua del mate para calmar el dolor de cabeza.

Spathicarpa hastifolia Hook. (Araceae), jety ta'ytetu. Sporolobus indicus (L.) R. Br. (Poaceae), kapi'i.

Stachytarpheta australis Moldenke (Verbenaceae), tatu ruguái. Las hojas en el mate se consideran estomacales y para dolores menstruales.

Stemodiaverticillata(Mill.)Hassl.(Scrophulariaceae), yvoty.

Stenachaenium riedelii Baker (Asteraceae), ambere ka'a. Con los tallitos sumergidos en el agua preparan un baño para las criaturas, que las preserva de todas las enfermedades

Stenandrium trinerve Nees (Acanthaceae), ypeku'i ka'a.

Strychnos niederleini Gilb. (Loganiaceae), ju'achĩ, mboguaju. Con las raspaduras de la raíz, cocidas con sal, se obtiene un líquido que se emplea en buches para curar las caries.

Styrax leprosus Hook. \& Arn. (Styracaceae), jaguarata'y, ka'a ovechĩ.

Syagrus romanzoffianum (Cham.) Glassman (Arecaceae), pindo. Con las fibras de las vainas foliares fabrican hilos que usan, entre otras cosas, para asegurar las puntas de flechas a la caña. Comen las frutas y el cogollo hervido: "pindo ru'a". Con el tronco ahuecado fabrican tambores "angu'a pu", usando como parches piel de agutí o tateto.

Talinum racemosum (L.) Rohrb. (Portulacaceae), guachu rembi'u, ka'a ruru, poã ro'y. Las hojas machacadas en agua fría se beben como refrescante cuando hace mucho calor; en baños se usa contra la fiebre.

Terminalia triflora (Griseb.) Lillo (Combretaceae), guajuvira morochĩ. Con la corteza retorcida hacen piolas.

Tibouchina gracilis (Bonpl.) Cogn. (Melastomataceae), pyri.

Tillandsia sp. (Bromeliaceae), ka'i avachĩ, karaguata'i.

Tradescantia fluminensis Vell. (Commelinaceae), jachyta ka'a, kochi rembi'u. La hoja machacada con leche se usa en gotas contra el dolor de vista. La maceración de los brotes en agua fría se bebe en casos de cefalalgias.

Tradescantia sp. (Commelinaceae), tupãchy recha'i.

Tragia sp. (Euphorbiaceae), tarami'ara. Con la decocción de la raíz con sal se hacen buches para calmar el dolor de muelas.

Trema micrantha (L.) Blume(Celtidaceae), kurunjy'ũ, kurundy'y. Trozos de corteza se ponen en el agua del mate en casos de cefalalgias.

Trichilia catigua A. Juss. (Meliaceae), kachygua, katygua pytã. Con la decocción de la corteza tiñen de pardo-rojizo las tiras de takuapi con que fabrican los cestos; para ello se cocinan trozos de corteza hasta que el líquido se va concentrando, y luego pintan la corteza de takuapi usando un marlo a modo de pincel. Reconocen tres clases diferentes de catygua, una que permite pintar de pardo, otra de rojo y otra de amarillo.

Trichilia clausseni C. DC. (Meliaceae), paro chanto. Hacen morteros en los que ponen agua que luego beben para calmar los dolores del corazón. Hacen crucifijos que cuelgan en el cuello cuando tienen algún dolor físico o moral.

Trichilia elegans A. Juss. (Meliaceae), katyguamirĩ. Dos o tres trozos de corteza se dejan macerar en un litro de agua fría, y esta se bebe en casos de mordeduras de víboras. Con el mismo fin, la corteza se ata a las piernas con un trapo. La madera se emplea para arcos.

Tridens brasiliensis (Nees ex Steud.) Parodi(Poaceae), kapi'i y.

Urera baccifera (L.) Gaudich. (Urticaceae), pynõ guachu. La decocción de la raíz se considera diurética y vermífuga, y se usa luego de los partos para aventar gases. Con las fibras de la corteza retorcida hacen redes para pescar y antiguamente tejían con ellas el chiripá y las cunas para los niños. Para extraer las fibras se dejan secar los tallos durante dos días, luego se secan a mano y se retuercen.

Vassobia breviflora (Sendtn.) Hunz. (Solanaceae), guachu ky'yĩ. Dicen que la fruta es venenosa.

Verbena montevidensis Spreng. (Verbenaceae), vervena guachu. La infusión o la decocción de la planta se beben en frío contra los vermes intestinales y el dolor de cabeza. Ver Allophyllus edulis y Setaria sulcata.

Vernicia fordii (Hemsl.) Airy Shaw (Euphorbiaceae). Su semilla se denomina tũ'a. 
Vernonia brevifolia Less. (Asteraceae), pỹnuarẽ'i ka'a.

Vernonia sp. (Asteraceae), gajo ruguái. La planta se sapeca, se muele, y con la decocción se lavan heridas.

Vernonia sp. (Asteraceae), gajo'y.

Vernonia sp. (Asteraceae), jagua petỹ, petỹrã, jagua ka'a. La decocción de la raíz se toma fría en casos de dolores estomacales; los brotes en agua fría se usan en lavados para el dolor de cabeza.

Vernonia sp. (Asteraceae), ka'avo tory. La decocción se toma en casos de dolores hepáticos.

Vernonia sp. (Asteraceae), torypa.

Victoria cruziana A.D. Orb. (Nynpheaeaceae), yrupe.

Vitex sp. (Verbenaceae), tarumá. Los frutos son comestibles, pero no los emplean en su alimentación: lo hacen, según ellos, otros indios salvajes. Emplean la decocción de las hojas para lavarse la cabeza.

Wilbrandia ebracteata Cogn. (Cucurbitaceae), chanjáu rã, ta'ytetu manjy'o. La raíz en decocción se emplea en casos de dolor de apéndice y, en el mate, para calmar el dolor de estómago y contra fiebres palúdicas.

Xanthium cavanillesii Schouw (Asteraceae), ypoty takua, mandyju rã, jaguarete py. La hoja calentada a fuego y embadurnada con grasa de cuatí, sirve para masajear suavemente las hinchazones causadas por la papera. En caso de mordedura de víboras, se machacan las hojas y se ponen en agua fría tomándose una cucharada, usándose el resto en lavados.

Xanthium spinosum L. (Asteraceae), chypa cabaju. Los cogollos se ponen en el agua del mate, a fin de calmar el dolor de cabeza y evitar enfermedades.

Xylopia brasiliensis Spreng. (Annonaceae), yvyra katu. Comen las frutas. Con la decocción de la corteza se prepara una bebeida para los dolores del corazón y otras enfermedades.

Xylosma venosa N.E.Br. (Flacourtiaceae), ju'y ro.

Zanthoxylum caribaeum Lam. (Rutaceae), tembetary ũ. Mismo uso que Zanthoxylum fagara.

Zanthoxylum fagara (L.) Sarg. (Rutaceae), tembetary pytã. La decocción de las hojas y la corteza se usa para el lavado del cuerpo, para combatir el cansancio.
Zanthoxylum petiolare A. St.-Hil. \& Tul. (Rutaceae), tembetary chayju. Mismos usos que sus congéneres.

Zanthoxylum sp. (Rutaceae), tembetary, yryvu retyma La decocción de la corteza se bebe como calmante para el dolor del apéndice; la de las hojas, contra la fiebre y el sebo'í, y se emplea en lavados en caso de conjuntivitis. Las hojas molidas se ponen en agua fría, y esta se bebe como vermífugo.

Zanthoxylum sp. (Rutaceae), tembetary. La decocción de las hojas, en baños, se utiliza para calmar dolores musculares.

Zea mays L. (Poaceae), avachĩ, avachĩ pichinga.

\section{Conclusiones}

La lista de plantas que Martínez Crovetto registró en su trabajo de campo con los guaraníes alcanza un total de 511 fitónimos distribuidos en 476 taxa, incluyendo especies nativas, adventicias y cultivadas.

El rango de distribución de las especies sugiere que durante sus campañas etnobotánicas abarcó todos los sectores y distritos considerados por él en su obra sobre fitogeografía de Misiones (Martínez Crovetto, 1963). Además, aparecen algunos nombres de plantas que no crecen en Misiones.

\section{Bibliografía}

CADOGAN, L. 1992. Diccionario Mbya-GuaraníCastellano. Biblioteca Paraguaya de Antropología, Asunción, 17: 1-211.

GUASCH, A. \& D. ORTIZ. 1995. Diccionario Castellano-Guaraní, Guarani-Castellano. Sintáctico, fraseológico, ideológico. $12^{\circ}$ edición. C. E. P. A. G. Asunción. 826 pp.

KELLER, H.A. 2008. Etnobotánica de comunidades guaraníes de Misiones, Argentina. Valoración de la vegetación como fuente de recursos. Tesis de Doctorado en Recursos Naturales, Facultad de Ciencias Agrarias, Universidad Nacional del Nordeste. $282 \mathrm{pp}$.

MARTINEZ-CROVETTO, R. N. 1963. Esquema fitogeográfico de la Provincia de Misiones (República Argentina). Bonplandia 1 (3): 171-223.

Original recibido el 22 de mayo de 2012; aceptado el 26 de septiembre de 2012. 
